\title{
Third generation stellar models for asteroseismology of hot B subdwarf stars
}

\section{A test of accuracy with the pulsating eclipsing binary PG 1336-018 *}

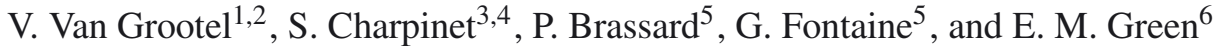 \\ ${ }^{1}$ Institut d'Astrophysique et de Géophysique, Université de Liège, 17 Allée du 6 Août, 4000 Liège, Belgium \\ e-mail: valerie.vangrootel@ulg.ac.be \\ 2 Chargé de recherches, Fonds de la Recherche Scientifique, FNRS, 5 rue d'Egmont, 1000 Bruxelles, Belgium \\ 3 Université de Toulouse, UPS-OMP, IRAP, 31028 Toulouse, France \\ ${ }^{4}$ CNRS, IRAP, 14 Av. E. Belin, 31400 Toulouse, France \\ 5 Département de Physique, Université de Montréal, CP 6128, Succursale Centre-Ville, Montréal, QC H3C 3J7, Canada \\ ${ }^{6}$ Steward Observatory, University of Arizona, 933 North Cherry Avenue, Tucson, AZ, 85721, USA
}

Received 12 December 2012 / Accepted 12 March 2013

\begin{abstract}
Context. Asteroseismic determinations of structural parameters of hot B subdwarfs (sdB) have been carried out for more than a decade now. These analyses rely on stellar models whose reliability for the required task needs to be evaluated critically.

Aims. We present new models of the so-called third generation $(3 \mathrm{G})$ dedicated to the asteroseismology of sdB stars, in particular to long-period pulsators observed from space. These parameterized models are complete static structures suitable for analyzing both $p$ - and $g$-mode pulsators, contrary to the former second generation $(2 \mathrm{G})$ models that were limited to $p$-modes. While the reliability of the $2 \mathrm{G}$ models has been successfully verified in the past, this important test still has to be conducted on the $3 \mathrm{G}$ structures.

Methods. The close eclipsing binary PG 1336-018 provides a unique opportunity to test the reliability of hot B subdwarf models. We compared the structural parameters of the sdB component in PG 1336-018 obtained from asteroseismology based on the 3G models, with those derived independently from the modeling of the reflection/irradiation effect and the eclipses observed in the light curve. Results. The stellar parameters inferred from asteroseismology using the 3G models are found to be remarkably consistent with both the preferred orbital solution obtained from the binary light curve modeling and the updated spectroscopic estimates for the surface gravity of the star. The seismology gives $M_{*}=0.471 \pm 0.006 M_{\odot}, R_{*}=0.1474 \pm 0.0009 R_{\odot}$, and $\log g=5.775 \pm 0.007$, while orbital modeling leads to $M_{*}=0.466 \pm 0.006 M_{\odot}, R_{*}=0.15 \pm 0.01 R_{\odot}, \log g=5.77 \pm 0.06$, and spectroscopy yields $\log g=5.771 \pm 0.015$. In comparison, seismology from a former analysis based on the $2 \mathrm{G}$ models gave very similar results with $M_{*}=0.459 \pm 0.005 M_{\odot}$, $R_{*}=0.151 \pm 0.001 R_{\odot}$, and $\log g=5.739 \pm 0.002$. We also show that the uncertainties on the input physics included in stellar models have, at the current level of accuracy, no noticeable impact on the structural parameters derived by asteroseismology.

Conclusions. The stellar models (both of second and third generation) presently used to carry out quantitative seismic analyses of sdB stars are reliable for the task. The stellar parameters inferred by this technique, at least for those that could be tested $\left(M_{*}, R\right.$, and $\log g$ ), appear to be both very precise and accurate, as no significant systematic effect has been found.
\end{abstract}

Key words. subdwarfs - stars: oscillations - stars: interiors - binaries: eclipsing - stars: individual: PG 1336-018

\section{Introduction}

Hot B subdwarf ( $\mathrm{sdB}$ ) stars are hot and compact objects with effective temperatures $T_{\text {eff }}$ between $20000-40000 \mathrm{~K}$ and surface gravities $\log g$ in the range 5.0 to 6.2 (see, e.g., Green et al. 2008). They occupy the so-called extreme horizontal branch (EHB), burning helium in the core and having a very thin residual hydrogen-rich envelope. The sdB stars dominate the population of faint blue objects down to $V \sim 16$ and are mainly found in the thick galactic disk, but also in the halo, as globular clusters members and in the galactic bulge (Altmann et al. 2004; Busso et al. 2005). Their presence is indirectly revealed in distant objects through their copious UV emission that is responsible for the UV-upturn phenomenon (an excess of UV emission)

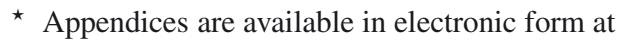
http://www . aanda.org observed, for instance, in old elliptical galaxies (Brown et al. 1997).

Major uncertainties remain about the details of the formation of the hot B subdwarfs. One important clue is the fact that the fraction of $\mathrm{sdB}$ stars in binaries with a stellar companion is found to be relatively high, around $\sim 50 \%$ (see, e.g., Allard et al. 1994; Maxted et al. 2001; Green et al. 2008). Plausible formation channels were modeled in detail from binary population synthesis by Han et al. (2002, 2003) and include binary evolution via a common envelope (CE), stable Roche lobe overflow (RLOF), and the merger of two helium white dwarfs, resulting in a single sdB star. The proposed scenario for forming single stars remains unsettled, however, as they could also result from single star evolution through enhanced mass loss at the tip of the red giant branch (RGB) at the moment of He-burning ignition (Dorman et al. 1993), or through a delayed He-flash, at higher effective temperatures during the collapsing phase after having left 
the RGB (D'Cruz et al. 1996). Another possibility is currently gaining observational support and involves the interaction of the star with close substellar companions (brown dwarfs and/or planets) in a common envelope evolution during the red giant phase. This scenario first proposed by Soker (1998) is strongly suggested by the discovery of small, nearly Earth-sized planets around the isolated sdB star KPD 1943+4058 (KOI 55) that could be remnants of former giant planets that survived engulfment in the red giant envelope and contributed to expelling the stellar envelope (Charpinet et al. 2011a). These distinct evolutionary scenarios leave a clear imprint on the binary fraction and distribution of sdB and their companion stars (CE evolution produces sdBs in very close binary systems preferably with white dwarf companions, RLOF gives rise to longer period $\mathrm{sdB}+$ main-sequence star binaries), but also and most importantly, on the stellar mass distribution for sdB stars. After core-He exhaustion, the sdB stars evolve directly toward the white dwarf cooling sequence as AGB-Manqué stars (Dorman et al. 1993).

Asteroseismology should help clarify the question of the formation of sdB stars. The sdB stars host two groups of nonradial pulsators. The first group, named the V361 Hya stars (originally EC 14026 stars; Kilkenny et al. 1997), exhibits short-period pulsations in the range 80 to $600 \mathrm{~s}$ with amplitudes of a few milli-magnitudes. The pulsations correspond to low-order lowdegree $p$-modes, which in sdB stars have significant amplitudes mainly in the outermost layers (Charpinet et al. 2000). The second group, discovered by Green et al. (2003) and referred to as V1093 Her stars, exhibits much longer periods from $45 \mathrm{~min}$ to a few hours. This corresponds to mid- and high-order low-degree $g$-modes, which can propagate in deep regions of the star, down to the convective He-burning core. Both $p$ - and $g$-mode pulsations are driven by the same $\kappa$-mechanism powered by local envelope accumulations of heavy elements such as iron due to radiative levitation (Charpinet et al. 1996, 1997; Fontaine et al. 2003; see also Hu et al. 2011). Quantitative asteroseismology of pulsating sdB stars has developed considerably during the past ten years, giving us new ways of revealing the properties of these objects at an unprecedented level of accuracy. This includes, among many other structural parameters, tight constraints on their masses. The results obtained so far are compiled in Table 1 of Fontaine et al. (2012), with the appropriate reference to each analysis that was carried out.

The method has long relied on stellar envelope models of the so-called second generation (2G) that incorporate the nonuniform abundance profiles of iron predicted by equilibrium between radiative levitation and gravitational settling (see Charpinet et al. 2002, for details). These models are suitable for asteroseismology of pure $p$-mode pulsators whose oscillation modes are sensitive only to the details of the outermost regions of the star. A very important test of accuracy for the seismic results obtained on the basis of these models was made using the star PG 1336-018 (Charpinet et al. 2008b). This object is one of the only two known sdB + dwarf $M$ close eclipsing binaries where the $\mathrm{sdB}$ component is a pulsating sdB star (the other one being 2M 1938+4603 recently discovered with Kepler; Østensen et al. 2010). The system PG 1336-018 permitted us to compare results obtained from the two independent techniques of asteroseismology and also orbital light curve and eclipse modeling. It was found that asteroseismology is remarkably consistent with one of the orbital solutions uncovered by Vučković et al. (2007). This close agreement showed that the seismic inference is both precise and accurate (i.e., free of significant systematics), at least for the stellar parameters that could be tested. This was of course an important finding attesting that the $2 \mathrm{G}$ models used for the quantitative asteroseismic studies of V361 Hya stars are sufficiently reliable for the task.

Recently, the advent of the space missions CoRoT and Kepler opened up new opportunities to gather sufficiently highquality data for the very low-amplitude, long-period $g$-mode pulsations seen in sdB stars for asteroseismological purposes. Three $g$-mode sdB pulsators have already been analyzed on this basis, providing for the first time information on their He-burning cores - including size and composition - along with the determination of other structural parameters (Van Grootel et al. 2010a,b; Charpinet et al. 2011b). Because the $2 \mathrm{G}$ envelope models are no longer suitable for quantitative analyses of $g$-mode pulsators, a third generation $(3 \mathrm{G})$ of stellar models for accurate evaluations of the $g$-mode pulsation periods had to be developed for that purpose and were used in the studies cited above. These are complete stellar structures (as opposed to the $2 \mathrm{G}$ envelope models) that include a detailed description of the central regions and, like their $2 \mathrm{G}$ counterparts, the nonuniform abundance profile of iron in the envelope predicted by microscopic diffusion.

While the $2 \mathrm{G}$ models passed the test of reliability for asteroseismology of $p$-mode $\mathrm{sdB}$ pulsators with the case of PG 1336-018, the new 3G models still have to be verified in a similar way. In the following section (Sect. 2), we discuss the need for parameterized models in quantitative asteroseismology, and we describe the new $3 \mathrm{G}$ models that we use for analyzing pulsation data obtained on hot B subdwarfs. In Sect. 3, we reanalyze the available data on PG 1336-018 with these 3G models in order to confront the seismic inferences obtained on the fundamental parameters of this star with those resulting from the former seismic analysis based on $2 \mathrm{G}$ models, and the independent orbital light curve analysis of Vučković et al. (2007). This test is important for linking older asteroseismic results obtained with $2 \mathrm{G}$ models with newer results based on $3 \mathrm{G}$ models in order to estimate potential systematics. We also carry out experiments in order to assess the importance of known model uncertainties on the stellar parameters determined by asteroseismology. A summary and conclusion are finally provided in Sect. 4.

\section{Third generation of sdB models for seismology}

\subsection{Background}

It may be worthwhile here to give a brief historical account of how our group came to the development of successive generations of models for the specific purpose of applying asteroseismological techniques to sdB stars. We consider first generation (1G) models those stellar equilibrium structures for sdB stars with a uniform metallicity. They include full evolutionary models such as those provided by Dorman et al. (1993) at a time when no pulsating sdB stars were known, and one of us (S.C.) started a Ph.D. thesis to investigate the asteroseismological potential of these stars (Charpinet 1999). The $1 \mathrm{G}$ models also include static structures made of an envelope (calibrated in part on the basis of the evolutionary models of Dorman) sitting on top of an inert ball. These envelope models allowed flexibility, in particular for investigating the effects of changing the metallicity on the overall stability of pulsation modes. It was thus found that uniform metallicity models (both evolutionary and static) are generally stable against pulsations, unless the metallicity is increased to unrealistically high values (Charpinet et al. 1996).

These investigations with $1 \mathrm{G}$ models identified a region of local instability associated with an opacity feature (the so-called Z-bump) even though the modes were globally stable. Given that sdB stars are very hot objects, and given that they are known 
to be chemically peculiar, radiative levitation was the obvious new ingredient to incorporate into a next generation of equilibrium structures. The idea was to verify if enough iron could accumulate in the driving region through radiative levitation and push some pulsation modes toward instability. Hence, we developed our second generation $(2 \mathrm{G})$ models, which are again static envelope structures, but which incorporate a nonuniform distribution of iron (a representative Z-bump element) as determined by microscopic diffusion. In detail, the iron profiles are obtained through detailed calculations of radiative forces under the assumption of an equilibrium between radiative levitation and gravitational settling (resulting from the combined action of the pressure gradient and electric fields). This is called hereafter diffusive equilibrium, disregarding at this stage other competing processes (see Sect. 3.5.1). The computation of the radiative accelerations themselves and of the equilibrium abundances of $\mathrm{Fe}$ are done following the procedure described in Chayer et al. (1995). These 2G models have been extremely successful at explaining the location of the $p$-mode sdB pulsators in the $\log g-T_{\text {eff }}$ plane as well as their nonadiabatic properties, and they have resisted the test of time remarkably well (Charpinet et al. 1997, 2001, 2006; Randall et al. 2012). The building assumption that the local abundance of $\mathrm{Fe}$ in a sdB star could be estimated by equating the effects of radiative levitation with those of gravitational settling was ultimately confirmed through detailed timedependent calculations (see, e.g., Fontaine et al. 2006; Charpinet et al. 2008a).

Our driving motivation in the early years of sdB seismology was to explain the very existence of pulsators and account for the observed ranges of excited periods in these stars. Hence, simple envelope structures (such as the $2 \mathrm{G}$ models) incorporating the appropriate details of the driving/damping zone proved to be perfectly suitable for investigating the nonadiabatic properties of pulsating sdB stars. At the same time, Charpinet (1999) carried out a very detailed analysis with the evolutionary models of Dorman et al. (1993), and came to the conclusion that simple envelope models (obtained by cutting off the central zone of Dorman's evolutionary models or by building them with the same constitutive physics) would also be quite useful for quantitative adiabatic asteroseismology as long as only $p$-modes were involved (Charpinet et al. 2002). In contrast, systematic effects (the periods are increasingly overestimated with increasing radial order, reaching differences of up to 10-20\%) appear when dealing with $g$-modes, which is not surprising given that the central region of a sdB model carries a significant weight in terms of period formation for those modes contrary to $p$-modes. The use of the $2 \mathrm{G}$ models thus allowed us to combine both adiabatic and nonadiabatic seismology to quantitatively investigate the properties of the $p$-mode pulsators. Our initial effort in that direction was the study of the star PG 0014+067 which led to the first successful seismic inferences on the global structural parameters of a sdB pulsator, including its total mass (Brassard et al. 2001). This was before the discovery of the cooler and lower gravity long-period $g$-mode pulsators in 2003. The need for complete stellar models (incorporating the important central region for $g$-modes) became apparent when sufficiently high-quality data on $g$-mode pulsators became available from space missions.

\subsection{Description}

The 2G models are envelope structures built under the assumption that the luminosity is constant throughout the entire envelope. This, it turns out, is an excellent approximation for He-core burning stars on the EHB, as can be seen in Fig. 2 of
Charpinet et al. (2000), for example. As a consequence, only three equations remain to define the structural properties of such an envelope, and the integration is carried out from the surface inward down, typically, to a fractional mass depth $\log q \equiv$ $\log \left(1-M(r) / M_{*}\right)=-0.1$. There are four primary parameters to specify a $2 \mathrm{G}$ model: the effective temperature $T_{\text {eff }}$, the surface gravity $\log g$, the total mass of the star $M_{*}$, and the mass contained in the H-rich envelope, usually expressed as a logarithmic fractional mass, $\log \left(M_{\text {env }} / M_{*}\right)$. The first two $\left(T_{\text {eff }}\right.$ and $\left.\log g\right)$ can be directly related to observable quantities obtained by spectroscopy.

In the case of the $3 \mathrm{G}$ models, these are now complete stellar structures assumed to be in strict thermal and mechanical equilibrium. Thus, the luminosity of such a static model is exactly balanced by core He burning (and the very small contribution of $\mathrm{H}$ burning at the base of the H-rich envelope). The four usual equations of 1D stellar structure (minus the timedependent term) are simultaneously solved with the help of a relaxation scheme. The physical conditions at the outer boundary are handled through a gray atmosphere scheme. A thin convection zone is usually created around the opacity peak in the envelope of a $\mathrm{sdB}$ model, but the flux carried by convection across that thin zone is quite small, and well below the base of the atmosphere (Fig. 1). When present, convection is handled through one or another of the various versions of the mixing-length theory. In the present application, convection is modeled in terms of the standard ML1 version of Böhm-Vitense (1958).

The constitutive physics incorporates the nuclear reaction rates of Caughlan \& Fowler (1988) for H and, particularly, He burning. Electron screening is handled through the prescription of Graboske et al. (1973). Since it was developed to model white dwarf stars as well, it also incorporates the neutrino production rates described by Itoh et al. (1996, and references therein), and conductive opacities, also made available by Itoh et al. (2008, and references therein). The radiative opacities are OPAL data of 1995, including additional tables for iron in a hydrogen background especially computed for our needs by Forrest Rogers in 1996 and briefly described in Charpinet et al. (1997). These special tables are essential for properly mapping the opacity profile of $\mathrm{Fe}$ associated with radiative levitation. As indicated above, the nonuniform distribution of Fe with depth is obtained under the assumption of a perfect equilibrium between radiative levitation and gravitational settling following the procedure described in Chayer et al. (1995). The equation of state (EOS) is an in-house construction covering a large domain of the temperature-density plane, sufficiently large to cover all types of stars, including white dwarfs. Three regimes are involved. For the low-density regions, a network of Saha equations is solved for a mixture of radiation and an almost ideal (including Coulomb corrections), nondegenerate, partially ionized gas composed of a mixture of $\mathrm{H}, \mathrm{He}, \mathrm{C}$, and $\mathrm{O}$ in various proportions as needed. In the region of partial ionization where nonideal and degeneracy effects are important, we use the EOS of Saumon et al. (1995) for $\mathrm{H}$ and He, an improved version of the EOS of Fontaine et al. (1977) for C, and an unpublished EOS for O developed at Université de Montréal in 1998. The third regime covers the high-density domain corresponding to the fully ionized plasma in the liquid and, ultimately, in the solid phase according to the basic physics described by Lamb (1974) and improved by Kitsikis et al. (2005). Efforts were made to smoothly bridge the different EOS surfaces across the boundaries of the different regimes. This is of particular importance for pulsation studies. Let us note, however, that the third regime covering the highdensity domain is not relevant here for sdB stars. Interpolation 


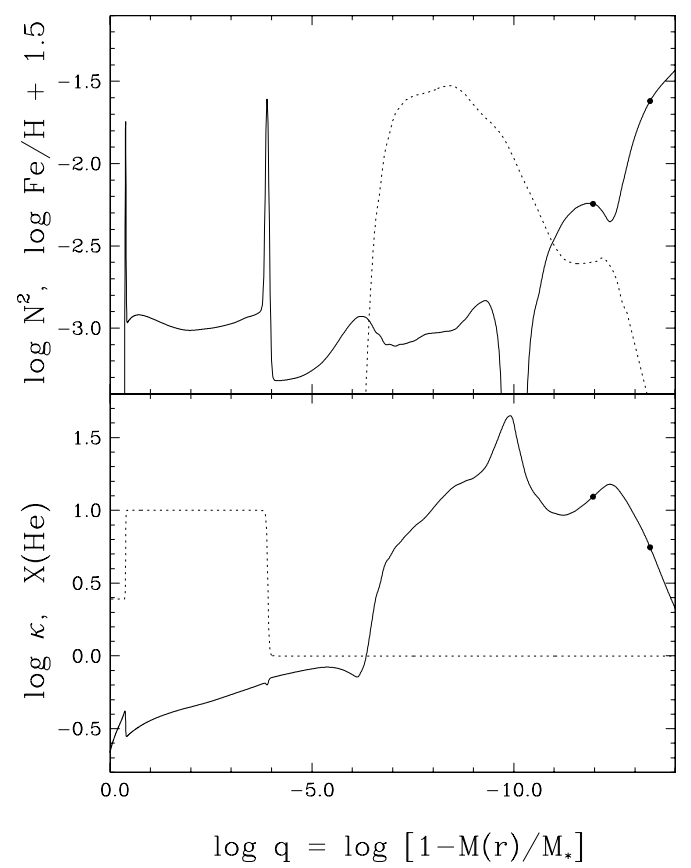

Fig. 1. Some structural properties for a representative $3 \mathrm{G}$ model. Upper panel: run of the logarithm of the square of the Brunt-Väisälä frequency as a function of fractional mass depth (solid curve) and equilibrium abundance profile of Fe (dotted curve, shifted upward by 1.5 dex). The solar ratio is $\log \mathrm{Fe} / \mathrm{H}=-4.5$ ( -3.0 on the shifted scale). Lower panel: run of the logarithm of the opacity as a function of depth (solid curve), and profile of the He distribution (dotted curve). In both panels the locations of the atmospheric layers, defined as those with an optical depth between $\log \tau=1.0$ and $\log \tau=-1.0$, are indicated by dots.

in composition is handled following the additive volume prescription of Fontaine et al. (1977).

The primary parameters to define a $3 \mathrm{G}$ model are the total mass of the star $M_{*}$, the mass contained in the outer H-rich envelope $\log \left(M_{\text {env }} / M_{*}\right)$, the mass contained in the (usually convective) core $\log \left(M_{\text {core }} / M_{*}\right)$, and the chemical composition in the core (with the constraint $X(\mathrm{He})+X(\mathrm{C})+X(\mathrm{O})+Z=1.0$ ). The stratified overall structure consists of a core, surrounded by a He-rich mantle, itself surrounded by a H-rich envelope in which Fe radiatively levitates under the assumption of diffusive equilibrium. The composition transition layers at the interface between the H-rich envelope and the He mantle, and those at the interface between the He-rich mantle and the core are modeled in terms of two additional parameters which become of relevance in presence of diffusion. In the present application, the composition transition zones have been calibrated on the basis of full evolutionary models such as those of Dorman et al. (1993), which do not take diffusion or possible sources of turbulence into account. These extra parameters are therefore held fixed in the present experiment (see, however, Sect. 3.5.2). Likewise, the value of $Z$ is fixed at 0 .

As an illustrative example, Fig. 1 depicts some of the structural properties of the $3 \mathrm{G}$ model identified below as the model that best accounts for the available seismic data on the pulsating sdB component of the PG 1336-018 system. The relatively sharp peaks which characterize the profile of the Brunt-Väisälä frequency near $\log q \simeq-0.35$ and $\log q \simeq-3.8$ are clearly associated with the abrupt change of chemical composition in the composition transition zones. The signature of these transition zones is also evident on the opacity profile, although the magnitude of that signature is much less important in that case. The model features a convective core and a much less significant convection zone in the outer envelope, less significant in the sense that it carries at most only $\sim 0.6 \%$ of the flux. This very weak convection zone is clearly associated with the maximum of the opacity profile, but does not change in any significant way the basic character of the driving mechanism, which remains essentially a pure $\kappa$-mechanism. It is the location and shape of the Fe opacity peak that provide the conditions for exciting $p$-mode pulsations in the model. Again, radiative levitation is the key element for boosting the abundance of $\mathrm{Fe}$ in the driving region, which is necessary for destabilizing pulsation modes. The opacity profile in the envelope affects not only the driving/damping processes, but also the adiabatic properties (particularly the pulsation periods for $p$-modes) through its direct effects on the mechanical structure of the model. Finally, this $3 \mathrm{G}$ model is a complete model and, therefore, provides a reliable equilibrium structure for deriving $p$-mode periods, and $g$-mode periods as well.

\subsection{The need for parameterized models}

When we started investigating the application of the forward asteroseismological method to pulsating hot subdwarf and white dwarf stars a decade ago (see, e.g., Brassard et al. 2001; or Fontaine \& Brassard 2002), we realized at the outset that the use of full evolutionary models would not be practical because it is nearly impossible, even with large computer clusters, to cover finely all of the relevant domains of parameter space with evolutionary sequences; it would take too much computing time. The practical consequence is that evolutionary sequences, necessarily limited in number, may actually miss the correct region of parameter space where the best seismic model resides.

As a fallback position, we turned to less realistic, static models of the kind described here. These allow maximum flexibility for very exhaustive searches in parameter space and the identification of a possible optimal seismic model. Of course, such a seismic model must ultimately be validated by detailed evolutionary calculations. The parameterized model approach can also be useful for readily identifying the dependences of the pulsation periods on specific model parameters. They can also help identify directly the parts of the input constitutive physics that may need improvement.

The parameterized static $3 \mathrm{G}$ models that we discuss in this paper are still crude models of hot subdwarf B stars. We believe, however, that they are defined in terms of the most sensitive parameters from a seismic point of view. It is important that they be tested as thoroughly as possible. Brassard \& Fontaine (2008) presented the results of a first test connecting the seismic model of PG 0014+067 derived from the $2 \mathrm{G}$ structures described by Brassard et al. (2001) with the new seismic solution based on the first $3 \mathrm{G}$ models that were being developed for that star. Interestingly and encouragingly, both solutions turned out to be essentially the same. A still more stringent test is described in the next section.

\section{Seismic test of accuracy for the new models}

\subsection{PG 1336-018: a Rosetta stone for asteroseismology}

The system PG 1336-018 (NY Virginis) is one of the very few known $\mathrm{sdB}+\mathrm{dM}$ close eclipsing binaries, and one of the only two known objects of this type where the sdB component is a pulsating star (Kilkenny et al. 1998). This makes PG 1336-018 the equivalent of a Rosetta stone permitting the direct comparison of independent techniques (namely orbital modeling, asteroseismology, and spectroscopy) used to derive some of the stellar 
parameters, and makes it possible to check the reliability of the methods and models, as well as the presence (or lack thereof) of biases in the derived parameters. This rare opportunity provides extremely important information on the accuracy actually achieved, as generally only the internal precision of the measurements given by the error estimates can be obtained.

Parameters for the two components of PG 1336-018 have been determined by combining multicolor ULTRACAM/VLT light curves and the radial velocity (RV) curve obtained from UVES/VLT spectra (Vučković et al. 2007). These authors modeled the reflection/irradiation effect and eclipses seen in the light curves and found three solutions - each deriving among other parameters a mass, a radius, and a surface gravity for the sdB star - of equal statistical significance, caused by the large parameter space and correlations between some parameters (see Table 2 for a summary of the relevant values). Independently, the first asteroseismic analysis of the $\mathrm{sdB}$ component carried out by Charpinet et al. (2008b) was based on the pulsation spectrum provided by Kilkenny et al. (2003). These authors identified 28 periodicities in the 96 to 205 s range by analyzing the multisite Whole Earth Telescope (WET) campaign held in April 1999, which constitutes the most extensive photometric monitoring available to date for this star. The seismic analysis was based on the $2 \mathrm{G}$ envelope models and yielded a solution with parameters falling very close to one of the solutions of Vučković et al. (2007), which derives for the sdB component a total stellar mass of $M_{*}=0.466 \pm 0.006 M_{\odot}$ and a radius of $R_{*}=0.15 \pm 0.01 R_{\odot}$, while the seismic analysis of the observed $p$-modes led to a total stellar mass of $M_{*}=0.459 \pm 0.005 M_{\odot}$ and a radius of $R_{*}=0.151 \pm 0.001 R_{\odot}$. This close agreement is within the $1 \sigma$ error estimates of each technique.

In the following, we present a new asteroseismic analysis of the sdB component of PG 1336-018 based on the same set of observed frequencies, but this time using the $3 \mathrm{G}$ complete models previously described. We also take this opportunity to update the spectroscopic estimates of the atmospheric parameters of PG 1336-018 reported in Charpinet et al. (2008b) on the basis of better spectra and of new NLTE model atmospheres including metals (Sect. 3.2; see also Table 1). Our goal is to provide a thorough comparison of the various results and test the validity of the $3 \mathrm{G}$ models for asteroseismology of sdB stars.

\subsection{Improved spectroscopy for PG 1336-018}

There are two reasons why we were prompted to reinvestigate this issue. First, in view of the high importance of PG 1336-018 as a testbed for stellar modeling and asteroseismology, we continued to obtain spectra over the orbital phase and ended up with a combined zero-velocity optical spectrum reaching a very high value of $S / N \sim 491$ around $4000 \AA$ (compared to $\sim 175$ in Charpinet et al. 2008b). We refer to that spectrum as Bok9 in what follows, reflecting the fact that it was gathered at the $2.3-\mathrm{m}$ Bok Telescope of the Steward Observatory Kitt Peak Station, and that it has a resolution of $8.7 \AA$ at $4000 \AA^{1}$. It is the sum of 14 individual spectra, 6 obtained when the reflection effect in the system was near minimum, and 8 taken at other orbital phases. We also combined the 6 former spectra into a single one named Bok9L (as in low reflection effect; $S / N \sim 344$ in the blue), and the 8 others to obtain Bok9H $(S / N \sim 350$ in the blue)

\footnotetext{
The spectrum named BG9 in Charpinet et al. (2008b) represents the sum of our first few spectra and is, therefore, part of Bok9.
}

in order to investigate the effects, if any, of the irradiation of the secondary on the derived atmospheric parameters.

The second reason is that we have now developed the capacity to build NLTE model atmospheres and synthetic spectra with arbitrary heavy element abundances for a wide range of $\mathrm{H}$ and He compositions within reasonable computation times. It is well known that hot B subdwarfs are all chemically peculiar stars and that their atmospheric compositions are nonstandard and vary from star to star. The effects of metals on the determination of the atmospheric parameters of sdB stars have been studied in the past (e.g., Heber et al. 2000), but this has been done only within the LTE approximation. In the present case, we use two of our recent NLTE model grids to investigate potential effects in the context of PG 1336-018.

The first grid is similar to the NLTE, H, and He model spectra used in Charpinet et al. (2008b), except that our new synthetic spectra extend out to the red to include the $\mathrm{H} \alpha$ region (not previously considered). The second NLTE grid includes a representative heavy element mixture inspired from the work of Blanchette et al. (2008). These authors presented a NLTE analysis of FUSE spectra to derive the abundances of several astrophysically important elements in the atmospheres of five typical long-period pulsating sdB stars. The five stars analyzed by Blanchette et al. (2008) show very similar abundance patterns, and it is from these results that we derived a representative composition using the most abundant heavy elements. Hence, we assumed atmospheres containing $\mathrm{C}$ (1/10 solar), $\mathrm{N}$ (solar), $\mathrm{O}$ (1/10 solar), Si (1/10 solar), S (solar), and Fe (solar). These two $3 \mathrm{D}$ grids $\left(T_{\text {eff }}, \log g\right.$, and $\left.\log N(\mathrm{He}) / N(\mathrm{H})\right)$ were computed with the public codes TLUSTY and SYNSPEC (Hubeny \& Lanz 1995; Lanz \& Hubeny 1995; Lanz et al. 1997). These codes have been parallelized by one of us (P.B.) to efficiently run on a computer cluster. With these two grids of synthetic spectra, as well as a corresponding LTE grid for comparison purposes, we analyzed the spectra Bok9, Bok9L, and Bok9H. We also reanalyzed the blue spectrum PB6, renamed Bok6 from then on, as it has a higher resolution $(\sim 6 \AA)$, but a somewhat lower $\mathrm{S} / \mathrm{N}$ of $\sim 80$.

In Table 1 we summarize the results of several analyses of the Bok6 and Bok9 spectra, including a test with a metalfree LTE grid for comparison purposes. For practical use (especially in the seismic investigation that follows), the solutions obtained with the NLTE grid including metals are to be preferred. Figures 2 and 3 show the spectral fit obtained with that grid for the Bok6 and Bok9 spectra, respectively. Given the exceptional $\mathrm{S} / \mathrm{N}$ ratio of the Bok9 spectrum, Fig. 3 reveals an extraordinarily good match, notwithstanding the He II $4686 \AA$ line (although we had to increase the vertical scale by a factor of 5 in that case otherwise the differences would not have been seen in the plot). Except for the redder regions where the $\mathrm{S} / \mathrm{N}$ has significantly decreased (specifically, near the $\mathrm{H} \alpha$ and He I $6678 \AA$ lines), the other weak features seen in the observed spectrum are real. For instance, the weak structures in the red wings of $\mathrm{H} \gamma, \mathrm{H} \delta$, and $\mathrm{H} \epsilon$ are due to metallic absorption of unknown origin. Likewise, the absorption structure seen in the red wing of He I $5876 \AA$ is also real and is due, in that case, to weak interstellar reddening associated with the well known Na I doublet. For our purposes, we will adopt the weighted means of the atmospheric parameters as inferred in Figs. 2 and 3. Hence our updated estimates of these parameters for the sdB component of PG 1336-018 are $T_{\text {eff }}=32807 \pm 82 \mathrm{~K}, \log g=5.771 \pm 0.015$ (in cgs units, as elsewhere in the text and in the tables), and $\log N(\mathrm{He}) / N(\mathrm{H})=$ $-2.918 \pm 0.089$. These estimates are very close to the former values adopted in Charpinet et al. (2008b), but are more precise. 
Table 1. Summary of all available atmospheric parameters of PG 1336-018 derived from spectroscopy.

\begin{tabular}{lccccc}
\hline \hline Spectrum & Type of model & $T_{\text {eff }}(\mathrm{K})$ & $\log g$ & $\log N(\mathrm{He}) / N(\mathrm{H})$ & Reference \\
\hline SAAO1 & LTE, H & $33139 \pm 1000$ & $5.78 \pm 0.10$ & $\ldots$ & Kilkenny et al. (1998) \\
SAAO2 & LTE, H & $32895 \pm 1000$ & $5.67 \pm 0.10$ & $\ldots$ & Kilkenny et al. (1998) \\
VLT/UVES & LTE, H, He & $31300 \pm 250$ & $5.60 \pm 0.05$ & $-2.93 \pm 0.05$ & Vucković et al. (2007) \\
PB6 & NLTE, H, He & $33220 \pm 170$ & $5.75 \pm 0.04$ & $-3.11 \pm 0.25$ & Charpinet et al. (2008) \\
BG9 & NLTE, H, He & $32380 \pm 150$ & $5.77 \pm 0.03$ & $-2.87 \pm 0.17$ & Charpinet et al. (2008) \\
Bok6 & NLTE, H, He & $32874 \pm 148$ & $5.705 \pm 0.028$ & $-3.066 \pm 0.250$ & This work \\
Bok6 & NLTE, H, He, metals & $33285 \pm 169$ & $5.707 \pm 0.028$ & $-3.016 \pm 0.216$ & This work \\
Bok9 & LTE, H, He & $31959 \pm 71$ & $5.823 \pm 0.018$ & $-2.847 \pm 0.101$ & This work \\
Bok9 & NLTE, H, He & $32359 \pm 84$ & $5.793 \pm 0.017$ & $-2.879 \pm 0.095$ & This work \\
Bok9 & NLTE, H, He, metals & $32649 \pm 97$ & $5.794 \pm 0.017$ & $-2.892 \pm 0.098$ & This work \\
Bok9H & NLTE, H, He, metals & $32608 \pm 104$ & $5.798 \pm 0.018$ & $-2.839 \pm 0.098$ & This work \\
Bok9L & NLTE, H, He, metals & $32748 \pm 111$ & $5.800 \pm 0.020$ & $-2.893 \pm 0.113$ & This work \\
\hline Former adopted value & NLTE, H, He & $32780 \pm 200$ & $5.76 \pm 0.03$ & $-2.94 \pm 0.14$ & Charpinet et al. (2008) \\
New adopted value & NLTE, H, He, metals & $32807 \pm 82$ & $5.771 \pm 0.015$ & $-2.918 \pm 0.089$ & This work \\
\hline
\end{tabular}

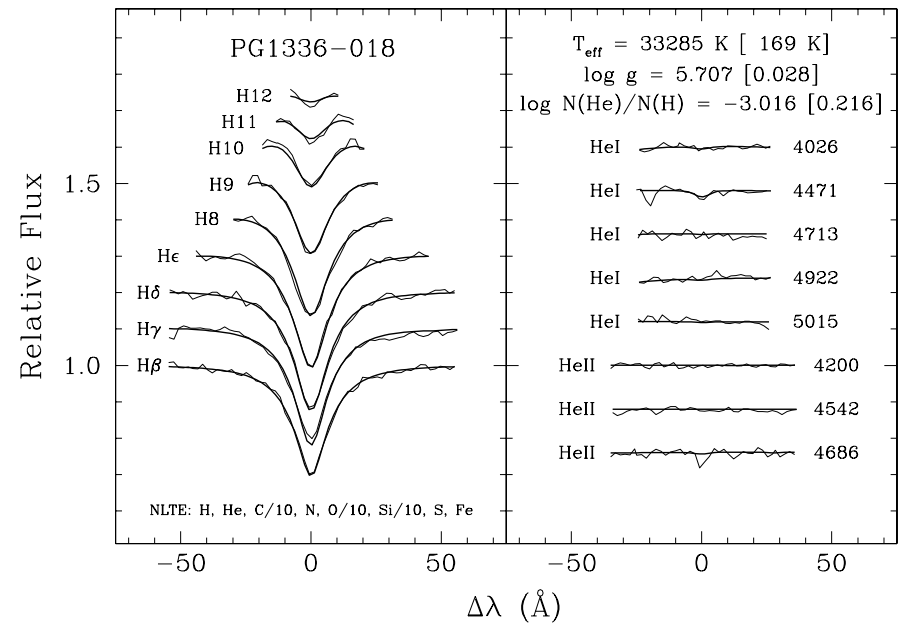

Fig. 2. Model fits (thick curves) to the hydrogen and helium lines (thin curves) available in our time-averaged, mid-resolution spectrum Bok6.

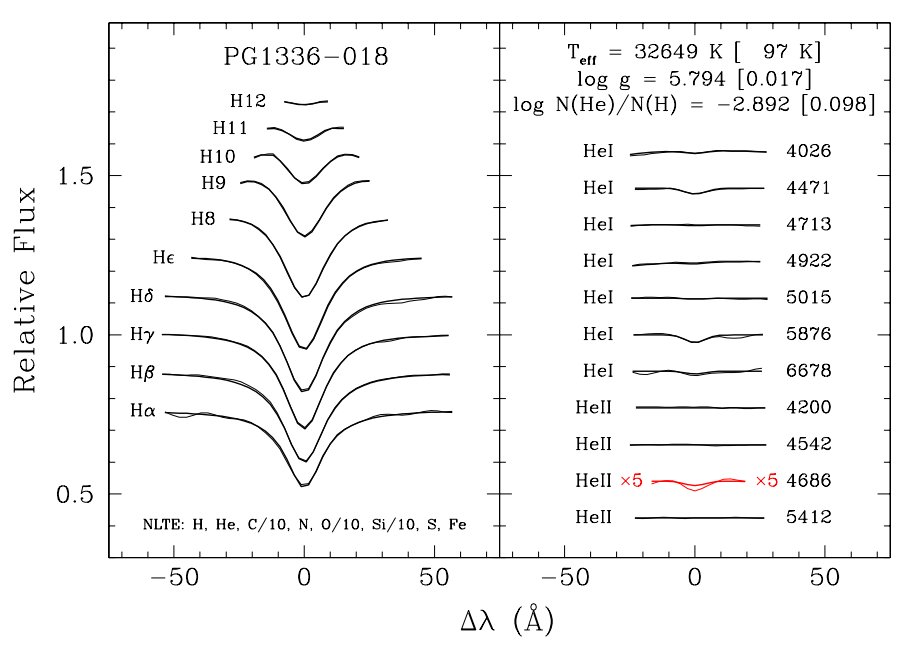

Fig. 3. Model fits (thick curves) to the hydrogen and helium lines (thin curves) available in our time-averaged, very high signal-to-noise, lowresolution spectrum Bok9.

aimed at isolating the model(s) that best fit the pulsation periods identified in this star. This is done by minimizing a $\chi^{2}$-type merit function of general form

$S^{2}=\sum_{i=1}^{N_{\mathrm{obs}}}\left(\frac{P_{\mathrm{obs}}^{i}-P_{\mathrm{th}}^{i}}{\sigma_{i}}\right)^{2}$,

where $N_{\text {obs }}$ is the number of observed periods and $\sigma_{i}$ a weight that can be associated to each pair of observed/computed periods $\left\{P_{\mathrm{obs}}^{i}, P_{\mathrm{th}}^{i}\right\}$ for a given model. The minimization is done both at the level of matching the periods and in model parameter space. We refer the reader to Charpinet et al. (2008b) for further details. The main difference lies in the fact that the stellar structures are now calculated using $3 \mathrm{G}$ models while in the previous study $2 \mathrm{G}$ envelope structures have been used for the analysis. This has two consequences. First, the natural parameters needed to specify a $3 \mathrm{G}$ model (and therefore the parameter space to explore) are different. As indicated above, these parameters are the total stellar mass $M_{*}$, the fractional mass of the outer H-rich envelope $\log \left(M_{\text {env }} / M_{*}\right)$ also referred to as $\log q(\mathrm{H})$, the fractional mass of the convective core $\log \left(M_{\text {core }} / M_{*}\right)$ or $\log q$ (core), and the chemical composition in the core (under
The procedure for deriving the structural parameters of the pulsating sdB star in PG1336-018 is the same as the one described in Charpinet et al. (2008b). It is a double optimization scheme 
the constraint $X(\mathrm{He})+X(\mathrm{C})+X(\mathrm{O})=1)$. Second, the external constraints provided by spectroscopy $\left(T_{\text {eff }}\right.$ and $\left.\log g\right)$ can no longer be used to define a priori the relevant range for the explored parameter space. This is because $T_{\mathrm{eff}}$ and $\log g$ are no longer natural parameters of the $3 \mathrm{G}$ stellar models, contrary to $2 \mathrm{G}$ envelope structures. Instead, these quantities now depend on the four parameters mentioned above and their values are known only a posteriori, after a model is converged. Since spectroscopic constraints are essential to guide the search for a meaningful asteroseismic solution in the vast parameter space and avoid the multiplication of inconsistent solutions, the adopted solution is to incorporate these constraints within the optimization procedure itself, by eliminating de facto during the search the models in parameter space that differ too much in $T_{\text {eff }}$ and/or $\log g$ from the spectroscopic values (by applying a correction factor to the $S^{2}$ value). In what follows we used the updated atmospheric values adopted in the previous section with a tolerance of $3 \sigma$ (see Table 1). This approach ensures by construction a fair consistency with spectroscopy but of course there is no guarantee, a priori, that a good period fit exists within these constraints.

The search for best-fit solutions was launched in the largest possible parameter space relevant for sdB stars, $0.30 \leq$ $M_{*} / M_{\odot} \leq 0.70,-5.0 \leq \log q(H) \equiv \log \left(M_{\text {env }} / M_{*}\right) \leq-2.0$, $-0.40 \leq \log q$ (core) $\equiv \log \left(1-M_{\text {core }} / M_{*}\right) \leq-0.15$, and $0 \leq$ $X_{\text {core }}(\mathrm{C}+\mathrm{O}) \leq 0.99$, where $X_{\text {core }}(\mathrm{C}+\mathrm{O})$ is the fractional part of carbon and oxygen in the convective core ${ }^{2}$. The constraints on $\log q(\mathrm{H})$ and $M_{*}$ rely on expectations from stellar modeling and various formation scenarios for hot B subdwarfs (see Han et al. 2002, 2003), whereas the range for the core size is loosely inspired by horizontal branch stellar evolution calculations (Dorman et al. 1993).

We assume that the PG 1336-018 system has reached full spin-orbit synchronism, such that the $\mathrm{sdB}$ component rotates as a solid body with $P_{\text {rot }}=P_{\text {orb }}=2.42438$ h (Kilkenny et al. 2000; Kilkenny 2011). This hypothesis relies on very firm grounds, as demonstrated by Charpinet et al. (2008b). The rotationally split pulsation modes are calculated within the first-order perturbative approach. The importance of higher-order perturbation effects and tidal deformation caused by the $\mathrm{M}$ dwarf companion have been also discussed in Charpinet et al. (2008b), showing that they do not alter significantly the asteroseismic analysis, at the precision of the current modeling.

As in Charpinet et al. (2008b), we use 25 out of the 28 periods listed in Table 4 of Kilkenny et al. (2003), leaving aside 3 periods $\left(f_{10}, f_{13}\right.$, and $\left.f_{8}\right)$ that may be spurious. All theoretical modes of degree $\ell=0,1,2$, and 4 are considered in the 90 to $230 \mathrm{~s}$ period range. Those with $\ell=3$ are explicitly excluded, having an extremely low visibility in the optical domain owing to cancellation effects because of their specific surface geometry (Randall et al. 2005). As an additional constraint, we also limited the association of the dominant modes $f_{1}$ to $f_{5}$ to $\ell \leq 2$, while keeping it open for the lower amplitude modes. This assumption simply filters out eventual, unconvincing solutions where some observed periods of highest amplitude would be associated to $\ell=4$ modes. This is fully justified in view of independent constraints on mode identification obtained from multicolor photometry for some bright sdB stars, from which all the dominant

\footnotetext{
2 We have found that theoretical periods are not very sensitive to the exact core composition of $\mathrm{C}$ and $\mathrm{O}$. Grouping $(\mathrm{C}+\mathrm{O})$ in one parameter facilitates and speeds up the optimization procedure. This is equivalent to choosing the mass fraction of helium left in the core as the free parameter, since $X_{\text {core }}(\mathrm{He})=1-X_{\text {core }}(\mathrm{C}+\mathrm{O})$.
}

modes so far have been identified to $\ell=0,1$, and/or 2 modes (see, e.g., Tremblay et al. 2006; Van Grootel et al. 2008).

Within the search domain specified, the multimodal optimization code (see Charpinet et al. 2008b for details) spotted a region of parameter space where, strictly speaking, two close but distinct families of models corresponding to minima of the merit function exist. The absolute minimum $\left(S^{2} \sim 4.81\right)$ has $M_{*}=0.4699 M_{\odot}, \log q(\mathrm{H})=-3.899, \log q$ (core) $=$ -0.384 , and $X_{\text {core }}(\mathrm{C}+\mathrm{O})=0.609$. The second slightly shallower minimum $\left(S^{2} \sim 5.04\right)$ has quite similar stellar parameters, with $M_{*}=0.4795 M_{\odot}, \log q(\mathrm{H})=-3.831, \log q$ (core) $=$ -0.239 , and $X_{\text {core }}(\mathrm{C}+\mathrm{O})=0.544$. With effective temperatures of $33038 \mathrm{~K}$ (best-fit model) and $33053 \mathrm{~K}$ (second-minimum model), and surface gravities of 5.7713 and 5.7772, respectively. Despite this distinction, Figs. 4 and 5 show that the two abovementioned families of solutions cannot be statistically disconnected. These maps are projections of the merit function (renormalized to one at its minimum and shown on logarithmic scale) on the 2-dimensional plane, i.e., at a given point of a map, the $S^{2}$ value is the minimum among all the values when the two other parameters are varied independently. The regions of the best-fit model and the second-minimum model are connected at the $2 \sigma$ level (white dotted contours). It is therefore appropriate to treat them as a unique family, adopting the absolute minimum as the model of reference that best match the periods observed in PG 1336-018. More information can be given on this best-fit model, including the details of the period match and mode identification, but since this is not essential for our purposes, we relegate this information to Appendix A. The only relevant point for the discussion is that the relative average dispersion achieved for this best fit model is $\overline{\Delta X / X}=0.18 \%$ (where $X$ is either the period $P$ or the frequency $v$ ), corresponding to $\overline{\Delta P}=0.30 \mathrm{~s}$ and $\overline{\Delta v}=11.4 \mu \mathrm{Hz}$. The average precision in simultaneously reproducing the observed periods of PG 1336-018 with the $3 \mathrm{G}$ models is very close to that achieved with the $2 \mathrm{G}$ models $(\overline{\Delta X / X}=0.17 \%, \overline{\Delta P}=0.27 \mathrm{~s}$, and $\overline{\Delta v}=10.5 \mu \mathrm{Hz}$; Charpinet et al. 2008b). Hence the optimal seismic $3 \mathrm{G}$ model is essentially as good as its $2 \mathrm{G}$ counterpart to that respect.

In Fig. 4, the solid and dotted vertical lines correspond to the three orbital solutions and their associated $1 \sigma$ uncertainties for the mass of the $\mathrm{sdB}$ component derived by Vučković et al. (2007): $0.389 \pm 0.005 M_{\odot}, 0.466 \pm 0.006 M_{\odot}$, or $0.530 \pm$ $0.007 M_{\odot}$. The right panel of Fig. 4 is a close-up view of the $M_{*}-\log q(\mathrm{H})$ plane in the region of interest. The valley of low $S^{2}$-values clearly points toward the second orbital solution of Vučković et al. (2007), as was already found with the $2 \mathrm{G}$ envelope models (Charpinet et al. 2008b). Figure 5 shows an elongated valley along the $\log q$ (core) parameter between -0.40 and -0.20 , which reflects the fact that the $p$-modes observed in PG 1336-018 are not sensitive to the stellar innermost layers, and therefore the size of the core cannot be constrained. The core composition is, however, indirectly constrained through its impact on the overall hydrostatic equilibrium of the star which affects the pulsation periods and the atmospheric parameters $\left(T_{\text {eff }}\right.$ and $\log g$ ) for a given stellar mass and envelope mass (see evolutionary tracks of sdB stars from, e.g., Dorman et al. 1993).

More quantitative statements can be made by statistically estimating the value for each parameter of PG 1336-018 and its associated error from the asteroseismic fit. We adopt here a new procedure differing from our former methods to estimate errors based on contour maps and $\chi^{2}$ significance levels (see, e.g., Charpinet et al. 2008b). We note, however, that the two approaches are essentially equivalent and provide quantitatively 

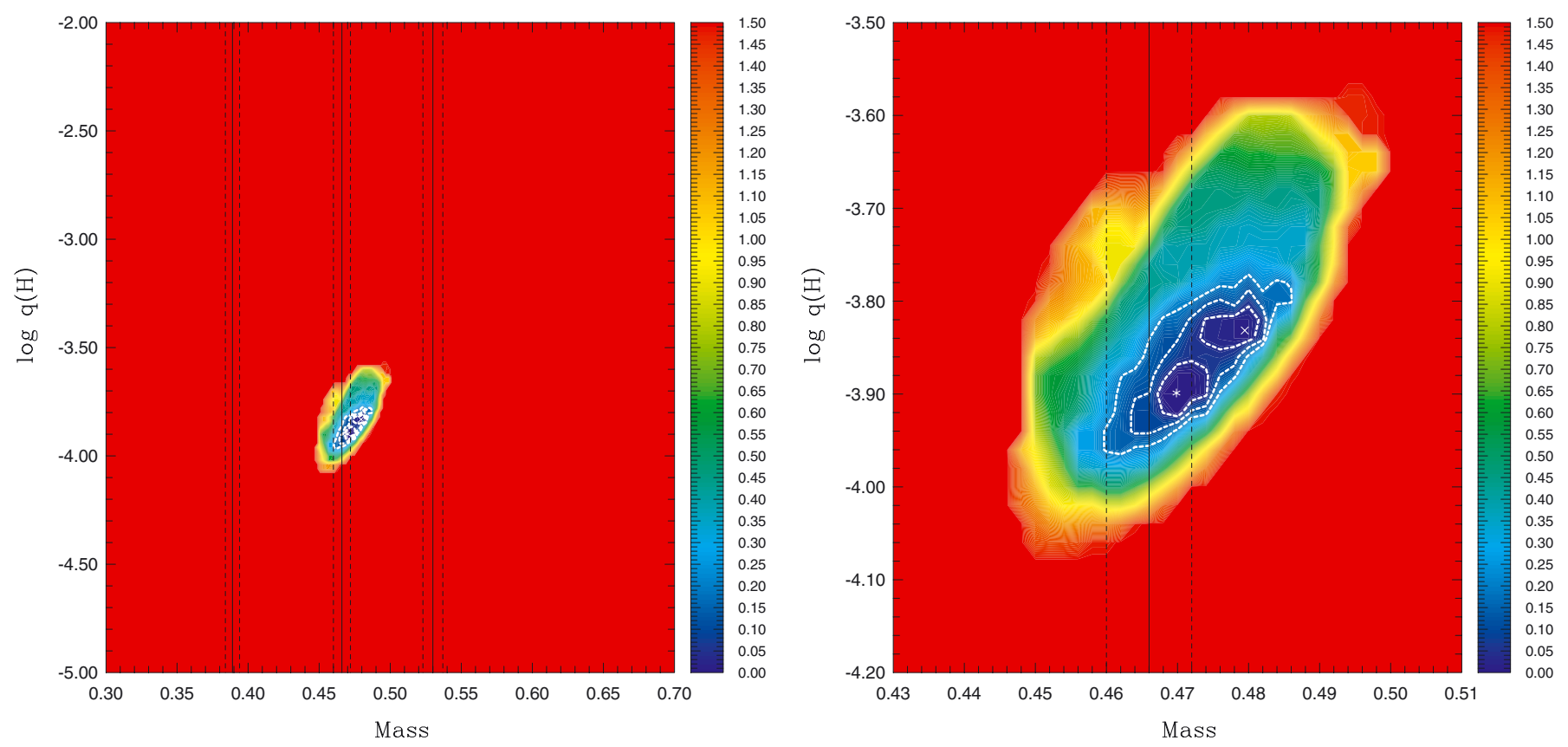

Fig. 4. Left panel: map of the projected merit function (on a $\log$ scale) along the $M_{*}-\log q(\mathrm{H})$ plane. Right panel: zoom of the left panel in the region of interest. At each $M_{*}, \log q(\mathrm{H})$ position, the value given is the projected merit function, i.e., the minimum of log $S^{2}$ that can be found among the values obtained for all $\log q($ core $)$ and $X_{\text {core }}(\mathrm{C}+\mathrm{O})$. The best-fit model is indicated by an asterisk, while the model corresponding to the second best-fit model is indicated by a cross. White contours show regions where the frequency fits have $S^{2}$ values within the $1 \sigma, 2 \sigma$, and $3 \sigma$ confidence levels relative to the best-fit solution.

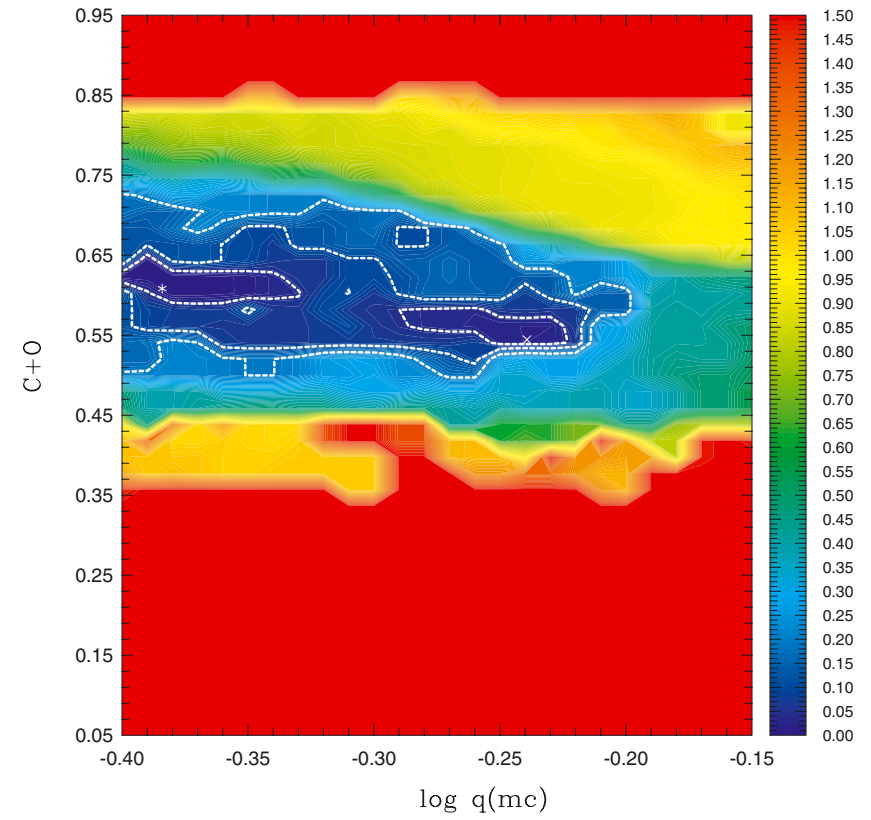

Fig. 5. Map of the projected merit function (in a log scale) along the $\log q($ core $)-X_{\text {core }}(\mathrm{C}+\mathrm{O})$ plane. At each $\log q($ core $), X_{\text {core }}(\mathrm{C}+\mathrm{O})$ position, the value given is the minimum of $\log S^{2}$ that can be found among the values obtained for all $M_{*}$ and $\log q(\mathrm{H})$. The best-fit model is indicated by an asterisk, while the model corresponding to the second minimum of the merit function is indicated by a cross. White contours show regions where the frequency fits have $S^{2}$ values within the $1 \sigma$, $2 \sigma$, and $3 \sigma$ confidence levels relative to the best-fit solution.

comparable error estimates (see below). We calculate the likelihood function

$\mathcal{L}\left(a_{1}, a_{2}, a_{3}, a_{4}\right) \propto \mathrm{e}^{-\frac{1}{2} S^{2}}$

from the $\chi^{2}$-type merit function $S^{2}\left(a_{1}, a_{2}, a_{3}, a_{4}\right)$ that was sampled by the optimization code during the search for the best-fit models along with additional grid calculations covering the regions of interest in parameter space. For each parameter of interest, say $a_{1}$, this function is integrated over the full parameter range covered by the other free parameters, thus defining a density of probability function for the chosen parameter

$\mathcal{P}\left(a_{1}\right) \mathrm{d} a_{1} \propto \mathrm{d} a_{1} \iiint \mathcal{L}\left(a_{1}, a_{2}, a_{3}, a_{4}\right) \mathrm{d} a_{2} \mathrm{~d} a_{3} \mathrm{~d} a_{4}$.

This density of probability function is then normalized assuming that the probability is equal to 1 that the value of $a_{1}$ is in the range specified for the search of a solution. In other words, the normalization factor is such that

$\int \mathcal{P}\left(a_{1}\right) \mathrm{d} a_{1}=1$,

over the allowed parameter range. This method permits the construction of histograms for the probability distribution of each primary model parameter. Secondary model parameters, like $T_{\text {eff }}, \log g$, the radius $R$, and the luminosity $L$ can also be evaluated in a similar way. Let $b_{1}$ be one of these secondary parameters. The density probability function is then

$$
\mathcal{P}\left(b_{1}\right) \mathrm{d} b_{1} \propto \mathrm{d} b_{1} \iiint \int_{b \in\left[b_{1}, b_{1}+\mathrm{d} b_{1}\right]} \mathcal{L}\left(a_{1}, a_{2}, a_{3}, a_{4} ; b\right) \mathrm{d} a_{1} \mathrm{~d} a_{2} \mathrm{~d} a_{3} \mathrm{~d} a_{4},
$$

where the integration is done with the additional constraint that for a given set of primary parameters, the corresponding value $b$ of the secondary parameter considered must be within $b_{1}$ and $b_{1}+\mathrm{d} b_{1}$. Again, the density of probability for the secondary parameters is normalized such that

$$
\int \mathcal{P}\left(b_{1}\right) \mathrm{d} b_{1}=1 \text {. }
$$

Figure 6 shows the histogram obtained for the probability distribution of the total mass of PG 1336-018 inferred from asteroseismology following the procedure described above. The 
V. Van Grootel et al.: Third generation stellar models for asteroseismology of hot B subdwarf stars

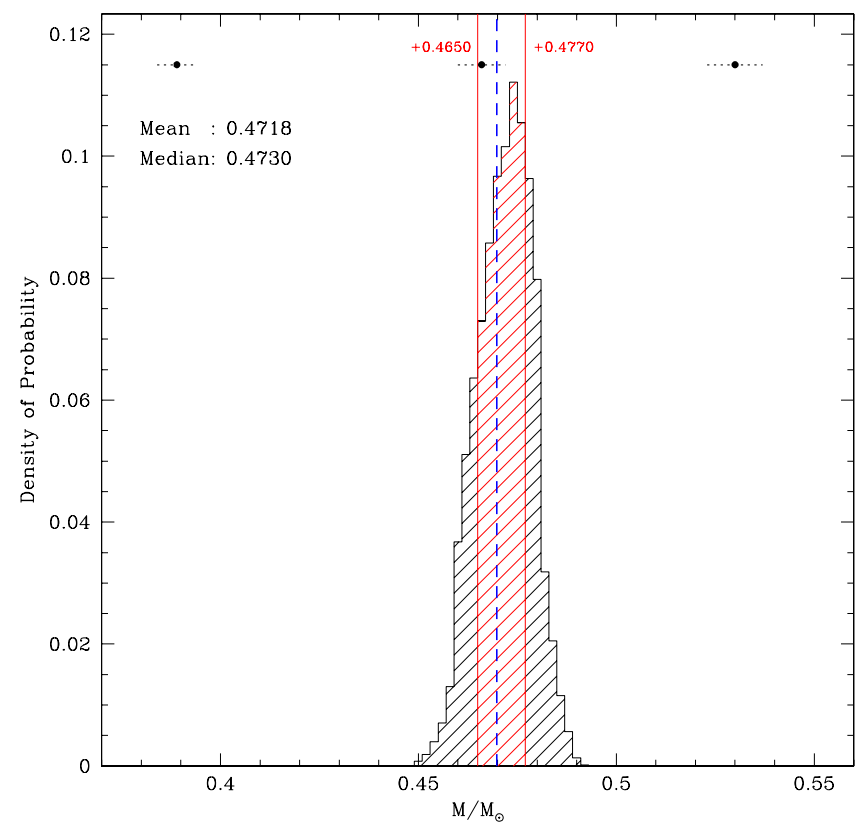

Fig. 6. Probability density function for the stellar mass from asteroseismology. The filled circles with the dotted lines are the three orbital solutions for the mass of the sdB component proposed by Vučković et al. (2007) with their $1 \sigma$ uncertainties. The red-hatched region between the two vertical solid red lines defines the $1 \sigma$ range, containing $68.3 \%$ of the mass distribution. The blue vertical dashed line indicates the mass of the optimal model solution of the lowest $S^{2}$-value.

range shown in this diagram only covers the region of interest, where a peak in the distribution is found (there is nothing below $0.37 M_{\odot}$ and above $0.56 M_{\odot}$ ) and where the three orbital solutions for the mass of the $\mathrm{sdB}$ component derived by Vučković et al. (2007) can be represented (filled circles with a horizontal dotted line indicating the $1 \sigma$ error). We find a very striking correspondence between the mass value derived from asteroseismology based on the new $3 \mathrm{G}$ models and the second orbital solution from Vučković et al. (2007). The two other orbital models giving respectively a much lower mass or a much higher mass for the sdB component are totally inconsistent with the seismic models and can be clearly excluded. From the histogram of the probability distribution, we can draw a statistical inference for the mass of the sdB star. The region between the two vertical lines (in red) contains $68.3 \%$ of the probability distribution and therefore defines, by convention, the $1 \sigma$ range for the value of the represented parameter. This range covers from $0.465 M_{\odot}$ to $0.477 M_{\odot}$, or equivalently we infer that, statistically, $M_{*}=0.471 \pm 0.006 M_{\odot}$ for the sdB star in PG 1336-018, taking the central value of the $1 \sigma$ range as the reference for simplicity. We recall that the relevant orbital solution of Vučković et al. (2007) yielded $M_{*}=0.466 \pm 0.006$ and that the seismic solution obtained by Charpinet et al. (2008b) based on $2 \mathrm{G}$ models led to $M_{*}=0.459 \pm 0.005$. Interestingly, the mean and median values of the mass distribution are equal to $0.472 M_{\odot}$ and $0.473 M_{\odot}$, respectively. The small differences in these values indicate that the distribution is close to, but not exactly, a normal (Gaussian) distribution, as can be easily seen in Fig. 6. The small visible distortion is to be related to the shape of the $S^{2}$ function illustrated in the right panel of Fig. 4, for instance. Finally, we note that the best-fit seismic model (of minimum $S^{2}$-value) shown as a vertical blue dashed line lies within the $1 \sigma$ range of the derived statistical mass distribution, meaning that this optimal model is not an outlier of the distribution and is representative of the star, as far as this parameter is concerned.

In the spirit of confronting the results of asteroseismology with other techniques, two additional parameters are of specific interest. First, the probability distribution obtained for the surface gravity $\log g$ (Fig. 7) can be compared with both the values derived from orbital modeling and spectroscopy. This distribution shows a very narrow peak with a $1 \sigma$ interval (containing 68.3\% of the distribution) between 5.768 and 5.781, or a statistically inferred value of $\log g=5.775 \pm 0.007$ (taking the center of the $68.3 \%$ significance interval as the reference value). The comparison with other determinations shows a perfect agreement, within the $1 \sigma$ errors of each technique, although the value determined from asteroseismology is much more precise. In particular we recall that the relevant orbital solution gives $\log g=5.77 \pm 0.06$ (filled triangle and horizontal dashed line in Fig. 7) and the spectroscopic analysis (Sect. 2) leads to $\log g=$ $5.771 \pm 0.015$ (filled circle and horizontal solid line in Fig. 7). The former asteroseismic solution obtained with $2 \mathrm{G}$ models gave $\log g=5.739 \pm 0.002$ (Charpinet et al. 2008b), also consistent with the other techniques (at the achieved precision), but somewhat shifted compared to the new estimate based on $3 \mathrm{G}$ models. Of course, the spectroscopic value was used to constrain the search of an asteroseismic solution. However, Fig. 7 shows that the adopted $3 \sigma$ tolerance for this constraint (dotted horizontal line) is much larger than the obtained probability distribution, indicating that the search was not overly constrained by spectroscopy. We also checked that relaxing the spectroscopic constraints further does not change the location and shape of this distribution at all. Finally, we note that the mean and median values of the distribution (5.775 and 5.776, respectively) are essentially equal and the derived asteroseismic values for $\log g$ are normally distributed to a very good approximation. Moreover, the $\log g$ value for the optimal model (the vertical dashed line in blue) is representative of the distribution, as is found within the $1 \sigma$ range.

The other interesting parameter for comparison purposes is the radius $R$ of the star (Fig. 8) derived from both asteroseismology and the analysis of the orbital light curve of the system (in particular the eclipses). The distribution for $R$ also shows a narrow peak with a $1 \sigma$ interval (containing $68.3 \%$ of the distribution) between $0.1465 R_{\odot}$ and $0.1483 R_{\odot}$, or a statistically inferred value of $R=0.1474 \pm 0.0009$ (taking the center of the $68.3 \%$ significance interval as the reference value). This is a very precise estimate which is in total agreement with the value derived for the relevant orbital solution of Vučković et al. (2007), which is, however, one order of magnitude less precise with an estimated value of $R=0.15 \pm 0.01 R_{\odot}$. The previous asteroseismic solution obtained with $2 \mathrm{G}$ models led to $R=0.151 \pm 0.001$ (Charpinet et al. 2008b), also consistent with the orbital value, but shifted notably (given the high-precision measurements) compared to the new estimate based on $3 \mathrm{G}$ models. Again, we note that the mean and median values of the distribution $(0.1474$ and 0.1475 , respectively) are nearly equal and the statistically derived asteroseismic value for $R$ essentially follows a normal distribution. We also note that the radius of the optimal model (the vertical dashed line in blue) is representative of this distribution (as it is within the $1 \sigma$ range).

Asteroseismology provides much more information on the star than the three parameters discussed above. For completeness, in Fig. 9 we show the histograms obtained for the probability distributions of the other derived parameters. In a nutshell, Fig. 9 shows that several parameters, namely the mass of the H-rich envelope $\log q(H)$, the mass fraction of $\mathrm{C}+\mathrm{O}$ in the 
Table 2. Structural parameters of PG 1336-018 derived from asteroseismology, spectroscopy, and orbital light curve analysis.

\begin{tabular}{|c|c|c|c|c|c|c|}
\hline \multirow[t]{3}{*}{ Quantity } & \multicolumn{2}{|c|}{ Asteroseismology } & \multirow{3}{*}{$\begin{array}{c}\text { Spectroscopy } \\
\text { Misc. } \\
\text { (see Sect. 3.2) }\end{array}$} & \multirow{2}{*}{\multicolumn{3}{|c|}{$\frac{\text { Orbital light curve modeling }}{\text { Vučković et al. (2007) }}$}} \\
\hline & \multirow{2}{*}{$\begin{array}{l}\text { This study } \\
\text { (3G models) }\end{array}$} & \multirow{2}{*}{$\begin{array}{c}\mathrm{C} 2008^{\dagger} \\
\text { (2G models) }\end{array}$} & & & & \\
\hline & & & & Model I & Model II & Model III \\
\hline$M_{*} / M_{\odot}$ & $0.471 \pm 0.006(1.3 \%)$ & $0.459 \pm 0.005$ & $\ldots$ & $0.389 \pm 0.005$ & $0.466 \pm 0.006$ & $0.530 \pm 0.007$ \\
\hline$R / R_{\odot}$ & $0.1474 \pm 0.0009(0.6 \%)$ & $0.151 \pm 0.001$ & $\ldots$ & $0.14 \pm 0.01$ & $0.15 \pm 0.01$ & $0.15 \pm 0.01$ \\
\hline $\log g$ & $5.775 \pm 0.007(0.1 \%)$ & $5.739 \pm 0.002$ & $5.771 \pm 0.015$ & $5.74 \pm 0.05$ & $5.77 \pm 0.06$ & $5.79 \pm 0.07$ \\
\hline$T_{\text {eff }}(\mathrm{K})$ & $32850 \pm 175(0.5 \%)$ & $32740 \pm 400$ & $32807 \pm 82$ & $\ldots$ & $\ldots$ & $\ldots$ \\
\hline $\log N(\mathrm{He}) / N(\mathrm{H})$ & & & $-2.918 \pm 0.089$ & $\ldots$ & $\ldots$ & $\ldots$ \\
\hline $\log \left(M_{\mathrm{env}} / M_{*}\right)$ & $-3.83 \pm 0.06(1.6 \%)$ & $-4.54 \pm 0.07$ & $\ldots$ & $\ldots$ & $\ldots$ & $\ldots$ \\
\hline $\log \left(1-M_{\text {core }} / M_{*}\right)$ & unconstrained & $\ldots$ & $\ldots$ & $\ldots$ & $\ldots$ & $\ldots$ \\
\hline$X_{\text {core }}(\mathrm{C}+\mathrm{O})$ & $0.58 \pm 0.06(10 \%)$ & $\ldots$ & $\ldots$ & $\ldots$ & $\ldots$ & $\cdots$ \\
\hline $\log \left(L / L_{\odot}\right)$ & $22.9 \pm 0.6(2.6 \%)$ & $23.3 \pm 1.5$ & $\ldots$ & $\ldots$ & $\ldots$ & $\ldots$ \\
\hline$M_{V}\left(g, T_{\mathrm{eff}}, M_{*}\right)$ & $4.60 \pm 0.04(0.8 \%)$ & $4.49 \pm 0.04$ & $\ldots$ & $\ldots$ & $\ldots$ & $\ldots$ \\
\hline$d\left(V, M_{V}\right)(\mathrm{pc})$ & $571^{\star} \pm 35(6.1 \%)$ & $619 \pm 38$ & $\ldots$ & $\ldots$ & $\ldots$ & $\ldots$ \\
\hline$P_{\text {rot }}(\mathrm{s})^{\ddagger}$ & $8727.7823 \pm 0.0002$ & $\ldots$ & $\ldots$ & $\ldots$ & $\ldots$ & $\ldots$ \\
\hline$V_{\mathrm{eq}}\left(P_{\mathrm{rot}}, R\right)\left(\mathrm{km} \mathrm{s}^{-1}\right)$ & $73.9 \pm 0.5(0.6 \%)$ & $75.9 \pm 0.6$ & $\ldots$ & $\ldots$ & $\ldots$ & $\ldots$ \\
\hline$i\left(^{\circ}\right)$ & $\ldots$ & $\ldots$ & $\ldots$ & $80.67 \pm 0.06$ & $80.67 \pm 0.06$ & $80.67 \pm 0.06$ \\
\hline$V \sin i\left(\mathrm{~km} \mathrm{~s}^{-1}\right)$ & $72.9 \pm 0.5(0.6 \%)$ & $74.9 \pm 0.6$ & $<79^{*}$ & $\ldots$ & $\ldots$ & $\ldots$ \\
\hline
\end{tabular}

Notes. ${ }^{(\dagger)}$ From Charpinet et al. (2008b). ${ }^{(\star)}$ Corrected for a reddening of $E(B-V)=0.021 \pm 0.009$. The 2008 distance estimate was not corrected for reddening. ${ }^{(\ddagger)}$ Synchronized at the orbital period value (from Kilkenny 2011). ${ }^{(*)}$ From Geier et al. (2010).

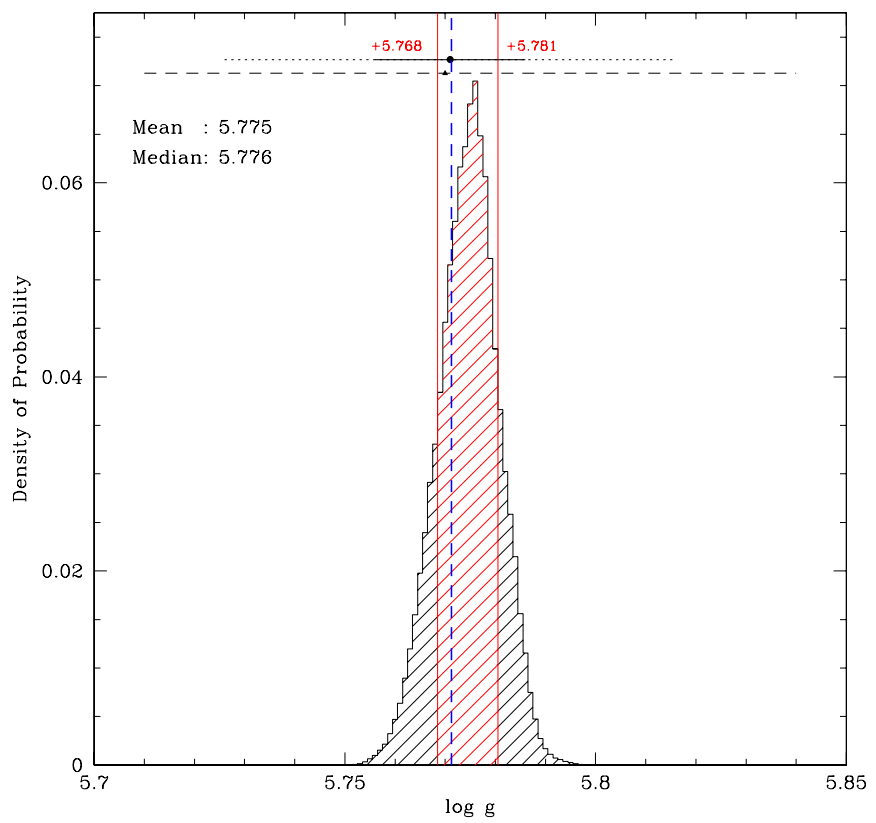

Fig. 7. Probability density function for the surface gravity $\log g$ from asteroseismology. The filled circle with the solid line and dotted line indicate the value derived from spectroscopy with its $1 \sigma$ and $3 \sigma$ uncertainties, respectively. The red-hatched region between the two vertical solid red lines is the $1 \sigma$ range, containing $68.3 \%$ of the $\log g$ distribution. The blue vertical dashed line corresponds to the $\log g$ value of the optimal model solution of the lowest $S^{2}$-value.

core $X_{\text {core }}(\mathrm{C}+\mathrm{O})$ (or equivalently the mass fraction of helium left there), and the luminosity of the star are well measured by asteroseismology at a very interesting precision (see Table 2 for the corresponding values and error estimates). It is important to recall that at least two of the above mentioned parameters cannot be measured by other means. For the three parameters discussed above, we also point out that the histograms shown in Fig. 9 are all close to normal (Gaussian) distributions. In contrast, the size of the mixed core $\log q$ (core) remains unconstrained, as the corresponding panel in Fig. 9 clearly shows. The distribution for this parameter is almost uniform and no useful information can

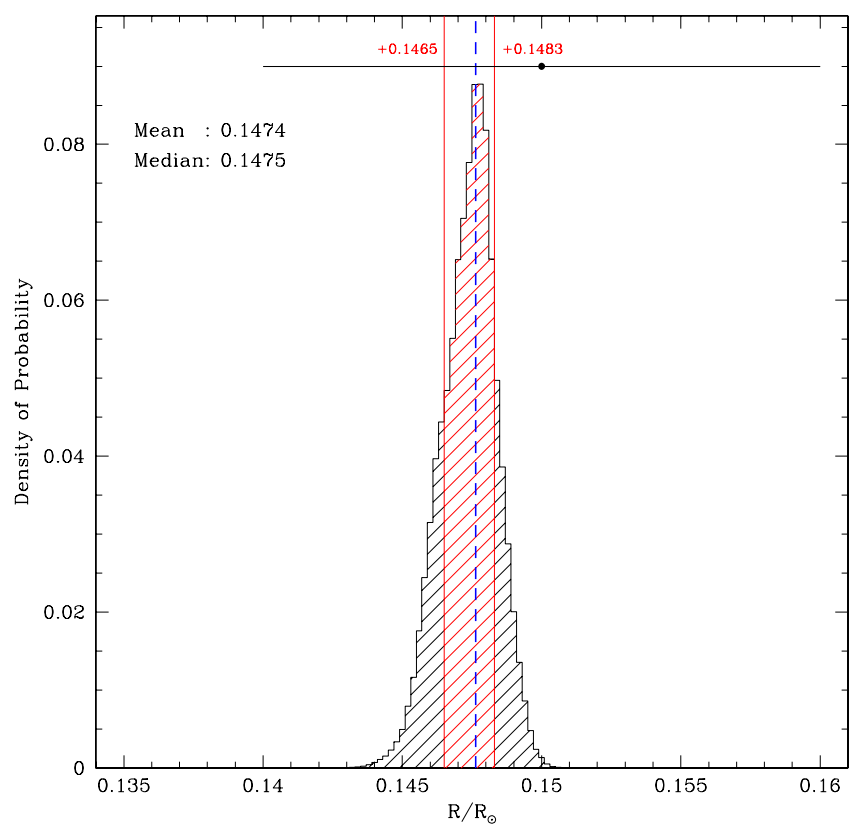

Fig. 8. Probability density function for the stellar radius from asteroseismology. The filled circle with the solid horizontal line indicates the $1 \sigma$ error associated to the relevant orbital solution from Vučković et al. (2007). The red-hatched region between the two vertical solid red lines is the $1 \sigma$ range containing $68.3 \%$ of the radius distribution. The blue vertical dashed line corresponds to the radius of the optimal model solution of the lowest $S^{2}$-value.

be obtained. This is not surprising since the $p$-modes observed in PG 1336-018 are essentially envelope modes that are largely insensitive to the detailed structure of the deepest regions, including in particular the core. It is only with $g$-mode pulsators that this quantity can be effectively measured. The last parameter is the effective temperature $T_{\text {eff }}$ which is known to be poorly measured by the $p$-modes in sdB stars (Charpinet et al. 2005). Indeed, in that case the corresponding panel in Fig. 9 shows that it is mainly the spectroscopic constraint imposed during the search for an asteroseismic solution that limits the range of the distribution. Finally, we stress that all the parameter values 
V. Van Grootel et al.: Third generation stellar models for asteroseismology of hot B subdwarf stars
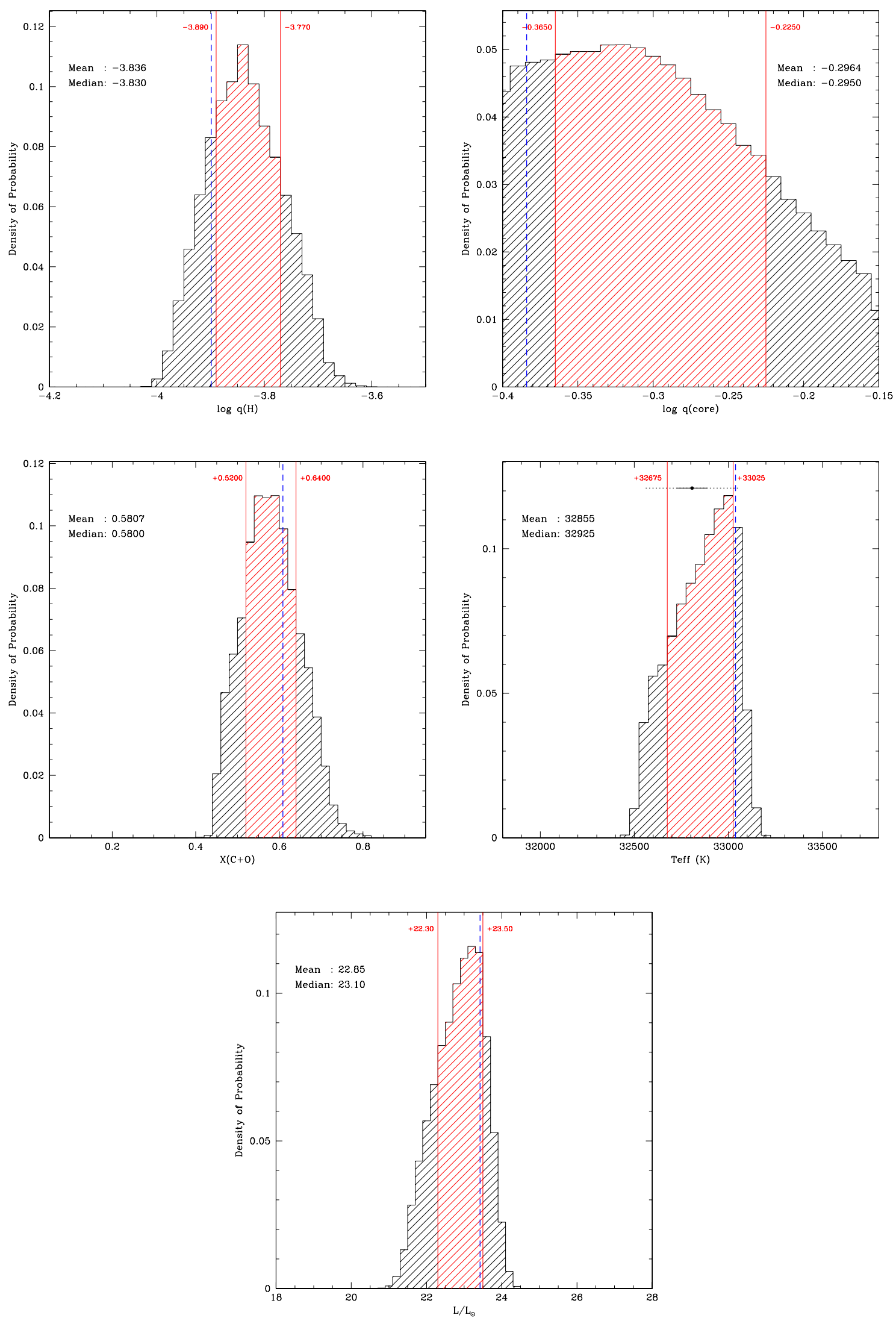

Fig. 9. Probability density functions derived from asteroseismology for the parameters $\log q(\mathrm{H}), \log q(\operatorname{core}), X_{\text {core }}(\mathrm{C}+\mathrm{O}), T_{\text {eff }}$, and $L_{*} / L_{\odot}$. The red-hatched regions between the two vertical solid red lines shows the $1 \sigma$ range containing $68.3 \%$ of the distribution. The blue vertical dashed line corresponds to the value of the optimal model. In the panel showing the distribution for $T_{\text {eff }}$, the filled circle is the spectroscopic value with its $1 \sigma$ (solid horizontal line) and $3 \sigma$ (dotted horizontal line) uncertainties. 
characterizing the optimal model that provides the absolute best match to the pulsation periods of PG 1336-018 fall within (or very close to) the $1 \sigma$ range of their corresponding statistical distributions (Figs. 6-9; blue vertical dashed line). It proves that this optimal model is not an outlier of the distribution of models that could potentially fit reasonably well the pulsation periods, but instead is a realization of the statistics which is representative of the hot B subdwarf in PG 1336-018 and can safely be considered as the seismic reference for this star.

\subsection{Comparison between various estimations}

We summarize in Table 2 all the values inferred for the parameters of PG 1336-018 from the various techniques employed. This sets the stage for a comparative discussion of the various results and an assessment of eventual systematic effects. As already mentioned, the best tested parameters are $M, R$, and $\log g$ because they have been measured quite precisely from both asteroseismology and orbital modeling, as well as from spectroscopy for the surface gravity. For these three parameters, all measurements indicate a particularly strong (within the $1 \sigma$ errors) consistency. Notably, the stellar mass is precisely and accurately determined from asteroseismology using the $3 \mathrm{G}$ models according to model II of Vučković et al. (2007). This consistency was already proven for the $2 \mathrm{G}$ models (Charpinet et al. 2008b), indicating that no important bias exists in the determination of this parameter from asteroseismology. This constitutes a very strong test demonstrating the reliability of the models (both $2 \mathrm{G}$ and $3 \mathrm{G}$ ) for this type of seismic analyses. This is also a crucial point for the project of building an empirical mass distribution of sdB stars from asteroseismology (Fontaine et al. 2012). Such empirical mass distributions are essential for clarifying the question of the formation of sdB stars, and unbiased measurements are of course a key factor.

A closer comparison of the two sets of seismic parameters derived from $2 \mathrm{G}$ and $3 \mathrm{G}$ models, respectively, shows that in practical terms no important difference exists between the two evaluations. The intrinsic precision of these measurements is very high by usual standards, but in general the differences between the two analyses remain below the $3 \sigma$ errors (although several parameters can differ by more than $1 \sigma$ ). There is one exception, $\log \left(M_{\mathrm{env}} / M_{*}\right)$, which shows a significant systematic effect, as this parameter is estimated to be somewhat larger with the $3 \mathrm{G}$ models. This is related to the presence of a realistic stellar core in $3 \mathrm{G}$ models, which has a small but noticeable impact on the frequencies of $p$-modes, especially for degrees $l=0$ (Charpinet et al. 2002). These small frequency shifts slightly modify mode trapping, which depends on the positions of the eigenfunction nodes relative to the envelope $\mathrm{He} / \mathrm{H}$ transition (Charpinet et al. 2000). Mode trapping therefore primarily determines the value of $\log q(\mathrm{H})$, which thus slightly but significantly changes from $2 \mathrm{G}$ to $3 \mathrm{G}$ models. Overall, if the improved $3 \mathrm{G}$ complete stellar models of sdB stars are now to be preferred for asteroseismology purposes, this comparison underlines that the former $2 \mathrm{G}$ envelope models already provided quite robust estimations of the structural parameters of PG1336-018. By extension, this considerably increases our confidence in the seismic parameters derived for all the other $p$-mode $\mathrm{sdB}$ pulsators based on these $2 \mathrm{G}$ models.

Finally, we point out that this reliability check for the 3G models using PG1336-018 as a privileged testbed remains partial in the sense that only $p$-modes are observed in this pulsating sdB star. An extension of this test to a $g$-mode pulsator in a close eclipsing binary may become possible with the $\mathrm{sdB}+\mathrm{dM}$ system 2M 1938+4603 recently discovered by the Kepler space mission (Østensen et al. 2010). Barlow et al. (2012) proposed an orbital solution from Kepler eclipse timings, assuming a perfectly circular orbit. The mass ratio and therefore the masses of the two components are, however, very sensitive to the eccentricity. The light curve modeling of 2M 1938+4603 still has to be carried out in a fully consistent way, i.e., by using additional multicolor photometry as Vučković et al. (2007) do. On the asteroseismology front, the sdB component of $2 \mathrm{M} \mathrm{1938+4603}$ exhibits an extremely rich pulsation spectrum of both $p$ - and $g$-modes. Moreover, the star is presumably a fairly fast rotator if synchronization is achieved (which is likely considering the very short orbital period of $3.024 \mathrm{~h}$ ). For $g$-modes that have periods comparable to the expected rotation period of the $\mathrm{sdB}$ star (if synchronized), the simple perturbative approach for computing the pulsation spectrum can no longer be applied. One must then investigate the problem with more sophisticated methods based either on the traditional approximation or on nonperturbative treatments to compute the effects of rotation on stellar pulsations (Reese et al. 2009; Ouazzani et al. 2012). This star therefore currently poses a difficult challenge for the seismic modeling, but could become exploitable in the future, as work being done in that direction progresses.

\subsection{Impact of known model uncertainties on the seismic inference of stellar parameters}

The static $2 \mathrm{G}$ and $3 \mathrm{G}$ models developed for detailed asteroseismic studies of sdB stars include a simplified treatment of the effects of diffusion. Diffusion, along with other potentially competing mixing mechanisms, is a process that can modify the chemical stratification inside the star over time. It is therefore more difficult to implement in a strategy based on static stellar structures and approximate ways of dealing with it must be used. Diffusion will most notably have two main effects. First, it will control the heavy metal abundance profiles in the envelope of a sdB star under the pressure of selective radiative forces (radiative levitation). Second, as time passes it will also modify the profile of the chemical transition at the interface between the He mantle and the H-rich envelope, making it smoother. We investigate below the impact of uncertainties associated with these two effects on the seismic determination of stellar parameters. We also investigate the impact of uncertainties on He-burning rates, namely, the triple- $\alpha$ and ${ }^{12} \mathrm{C}(\alpha, \gamma){ }^{16} \mathrm{O}$ nuclear reactions.

\subsubsection{Impact of the iron composition profile}

In the simplified approach used in our models, only iron is considered (as the main contributor to the Rosseland mean opacity) and the stratification adopted is assumed to be the profile computed at diffusive equilibrium, i.e., equilibrium between radiative levitation and gravitational settling. This approximation is both practical (in a hydrostatic modeling context) and physically justified because diffusion timescales are short in the relevant region of the envelope, thus presumably leading almost instantly (relative to the secular evolution timescale) to an equilibrium state (Fontaine et al. 2006; Charpinet et al. 2008a). Of course, in reality, diffusive equilibrium is never completely reached and other competing phenomena can potentially affect the metal distribution such as stellar winds, turbulent mixing (induced, e.g., by differential rotation), and/or double diffusive (thermohaline) convection due to $\mu$-gradient inversions. These effects add to the difficulty of incorporating diffusion in static models and are 
V. Van Grootel et al.: Third generation stellar models for asteroseismology of hot B subdwarf stars
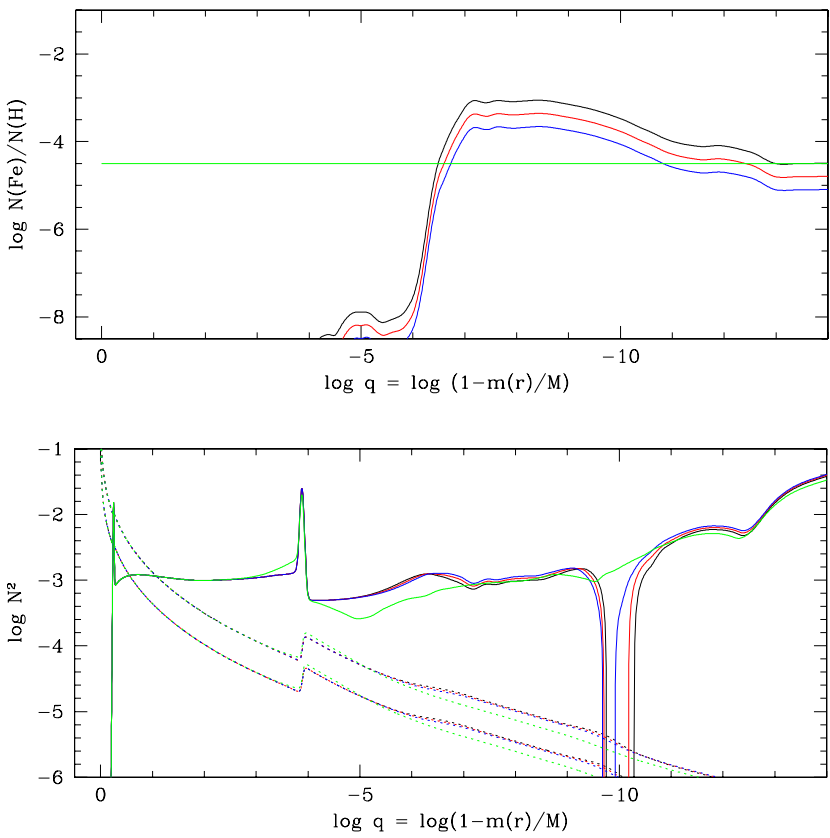

Fig. 10. Upper panel: iron abundance profiles used in four, otherwise identical, test models. The black, red, blue, and green curves correspond to the profile at diffusive equilibrium between gravitational settling and radiative levitation, the profile when the amount of levitating iron is decreased by a factor of two, the profile after a decrease of a factor of four, and the profile assuming a uniform iron distribution in solar proportion. Lower panel: corresponding profiles for the Brunt-Väisälä (solid curves) and Lamb (dotted curves for $l=1$ and $l=2$ ) frequencies.

quite uncertain in their details in evolutionary calculations as well. Therefore, strong uncertainties remain on the exact abundance distributions of chemical species inside the envelope of sdB stars. Because heavy elements (in particular iron) are the dominant source for the gas opacity, their distribution will affect the thermal structure of the star and consequently modify the periods of oscillation modes. However, this assessment has to be made more quantitative in order for the impact in the context of deriving structural parameters by asteroseismology to be truly appreciated. We demonstrate below that the uncertainty associated with the composition profiles built up by diffusion and by other processes in competition is not presently a strong issue when it comes to inferring structural parameters of sdB stars from their pulsation spectrum.

The first experiment is based on a set of representative $3 \mathrm{G}$ models built with the same input parameters but assuming different iron abundance profiles (see Fig. 10). The first model represents our standard $3 \mathrm{G}$ structures that use iron profiles expected at diffusive equilibrium. Two more models are constructed by artificially reducing the amount of levitating iron by a factor of two and four, respectively. A fourth model is built with a uniform iron abundance distribution in solar proportion. The purpose of this series of models is not to explore exhaustively all imaginable abundance profiles, which would of course be impossible to achieve. Instead, it allows us to sample a range of situations between two extreme cases where, on one hand, no diffusion occurs at all (the uniform solar model) and, on the other hand, full unperturbed diffusive equilibrium is reached (the standard model at diffusive equilibrium). We expect that the true profile is somewhere in between these two situations, the following results thus providing an accurate view of the magnitude of the induced effects in the context of asteroseismology.
Clearly, from the noticeable differences observed in the profiles of the Brunt-Väisälä and Lamb frequencies in the corresponding models (lower panel of Fig. 10), the different iron profiles should have some impact on the oscillation modes. Table 3 confirms this by providing the adiabatic periods of the lowdegree $(\ell=0-4) p$-modes computed for each of the four models illustrated in Fig. 10. The changes in the iron profile generate noticeable differences in periods between the standard model at diffusive equilibrium (dubbed $1 \cdot N(\mathrm{Fe}) / N(\mathrm{H})$ in Table 3 ) and the other models. These however remain relatively limited for models were the amount of levitating iron has been decreased by a factor of two and four, suggesting that the impact of the exact chemical composition in the sampled range should remain small. Not unexpectedly, the model with a uniform Fe profile in solar proportion shows larger variations, possibly leading to more significant systematic drifts in the model parameters derived from asteroseismology.

In order to quantify more precisely the real impact of these period changes, we reanalyzed the star PG 1336-018 following the same procedure as before (see Sect. 3.3), but using the various model assumptions about iron composition profiles discussed above. The results obtained with the standard 3G models (using an iron abundance profile at diffusive equilibrium) discussed in Sect. 3.3 are summarized for convenience in Col. 5 of Table 4. The probability density functions obtained for the main structural parameters of the star that depend on the abundance profiles assumed for iron in the stellar models are shown in Figs. B.1-B.3 of Appendix B. The corresponding statistical inferences for these parameters are given in Table 4. For each parameter, we also indicate in this table the largest systematic drift relative to the standard model (obtained by comparison with the model having a uniform and solar iron abundance) and the drift which is, in our view, more likely representative of the uncertainties associated with the metal abundance profiles in the envelope of sdB stars.

The main and obvious result of this exercise is that the overall impact of substantially changing the iron abundance profile is very small when it comes to inferring the structural parameters of the star from asteroseismology. Systematic effects on the derived values, when they exist, remain quite limited in amplitude relative to the current precision of the measurements. The most affected parameters are the radius with a drift of $-0.0068 \pm$ $0.0014 R_{\odot}(4.9 \sigma)$ in the worst case, but more reasonably a systematic effect of $-0.0031 \pm 0.0015 R_{\odot}(2.1 \sigma)$; the surface gravity $\log g$ with a drift of $+0.039 \pm 0.010 \operatorname{dex}(3.9 \sigma)$ in the worst case, and more reasonably $+0.020 \pm 0.011 \mathrm{dex}(1.8 \sigma)$; the H-rich envelope mass $\log q(H)$ with a drift of $-0.28 \pm 0.010 \operatorname{dex}(2.8 \sigma)$ in the worst case, but more reasonably no significant effect; and the luminosity $L$ with a drift of $-2.3 \pm 0.9 L_{\odot}(2.6 \sigma)$ in the worst case, and more reasonably $-1.1 \pm 0.9 L_{\odot}(1.2 \sigma)$. Of particular interest, we find that the mass determination does not suffer from any systematics at all (the value $+0.003 \pm 0.0011$ is consistent with no drift), thus strengthening the robustness of mass determinations of sdB stars based on asteroseismology. Consequently, the exact stratification of heavy metals that results from the competition of various diffusive and mixing processes occurring in the star and which is subject to large uncertainties is clearly not a strong issue at the currently achieved precision for the stellar parameters derived from asteroseismology.

Nevertheless, we point out that the test case of PG 1336-018 is further enlightening, because it provides a comparison with parameters independently derived from other techniques. Quite interestingly, Table 4 and Figs. B.1-B.3 show that our standard models assuming a nonuniform iron abundance profile at 
Table 3. Periods computed for a series of test models with various iron abundance, $\mathrm{He} / \mathrm{H}$ transition profiles, and modified nuclear reaction rates.

\begin{tabular}{|c|c|c|c|c|c|c|c|}
\hline \multicolumn{2}{|c|}{$\begin{array}{l}\text { Fe prof. }{ }^{a}: \\
\text { He/H prof. }{ }^{b}: \\
\text { Nucl. rates }\end{array}$} & $\begin{array}{c}1 \cdot \frac{N(\mathrm{Fe})}{N(\mathrm{H})}{ }^{\dagger} \\
\operatorname{sharp}^{\dagger} \\
\mathrm{CF}^{\dagger} 8^{\dagger}\end{array}$ & $\begin{array}{c}\frac{1}{2} \cdot \frac{N(\mathrm{Fe})}{N(\mathrm{H})} \\
\text { sharp } \\
\text { CF88 }\end{array}$ & $\begin{array}{c}\frac{1}{4} \cdot \frac{N(\mathrm{Fe})}{N(\mathrm{H})} \\
\text { sharp } \\
\text { CF88 }\end{array}$ & $\begin{array}{c}\text { Solar Fe } \\
\text { sharp } \\
\text { CF88 }\end{array}$ & $\begin{array}{c}1 \cdot \frac{N(\mathrm{Fe})}{N(\mathrm{H})} \\
\text { smooth } \\
\text { CF88 }\end{array}$ & $\begin{array}{c}1 \cdot \frac{N(\mathrm{Fe})}{N(\mathrm{H})} \\
\text { sharp } \\
2 \times \mathrm{CF} 88\end{array}$ \\
\hline$\ell$ & $k$ & \multicolumn{6}{|c|}{ Period (s) } \\
\hline 0 & 5 & 95.361 & 93.021 & 91.091 & 98.997 & 95.558 & 97.282 \\
\hline 0 & 4 & 110.383 & 107.854 & 105.720 & 114.996 & 110.289 & 112.633 \\
\hline 0 & 3 & 121.878 & 120.793 & 119.866 & 125.564 & 122.073 & 124.161 \\
\hline 0 & 2 & 143.687 & 139.815 & 136.578 & 152.200 & 143.704 & 146.740 \\
\hline 0 & 1 & 167.983 & 167.433 & 167.018 & 169.869 & 167.967 & 170.929 \\
\hline 0 & 0 & 181.622 & 180.359 & 179.550 & 215.586 & 181.500 & 185.170 \\
\hline 1 & 6 & 91.986 & 89.748 & 87.667 & 95.255 & 91.911 & 93.815 \\
\hline 1 & 5 & 101.879 & 100.347 & 99.033 & 104.349 & 102.002 & 103.844 \\
\hline 1 & 4 & 114.405 & 111.923 & 110.009 & 120.653 & 114.567 & 116.759 \\
\hline 1 & 3 & 138.376 & 135.678 & 132.900 & 142.634 & 138.265 & 141.102 \\
\hline 1 & 2 & 149.437 & 147.657 & 146.628 & 155.379 & 149.606 & 152.381 \\
\hline 1 & 1 & 180.242 & 178.677 & 177.624 & 214.649 & 180.120 & 183.837 \\
\hline 2 & 5 & 96.122 & 93.970 & 92.259 & 99.525 & 96.370 & 98.043 \\
\hline 2 & 4 & 111.296 & 108.611 & 106.408 & 116.728 & 111.270 & 113.597 \\
\hline 2 & 3 & 125.484 & 124.479 & 123.462 & 128.355 & 125.595 & 127.811 \\
\hline 2 & 2 & 144.146 & 140.426 & 137.428 & 152.362 & 144.254 & 147.202 \\
\hline 2 & 1 & 176.711 & 175.225 & 174.182 & 183.319 & 176.626 & 180.125 \\
\hline 2 & 0 & 186.021 & 185.753 & 185.606 & 213.268 & 186.069 & 189.270 \\
\hline 3 & 5 & 93.563 & 91.148 & 89.037 & 97.147 & 93.663 & 95.449 \\
\hline 3 & 4 & 106.995 & 104.923 & 103.075 & 110.121 & 106.922 & 109.112 \\
\hline 3 & 3 & 117.429 & 115.788 & 114.560 & 122.408 & 117.701 & 119.732 \\
\hline 3 & 2 & 141.849 & 138.076 & 134.813 & 149.620 & 141.908 & 144.840 \\
\hline 3 & 1 & 163.282 & 162.570 & 162.053 & 165.622 & 163.304 & 166.224 \\
\hline 3 & 0 & 180.063 & 178.710 & 177.833 & 210.379 & 180.184 & 183.593 \\
\hline 4 & 5 & 91.650 & 89.378 & 87.294 & 94.936 & 91.627 & 93.479 \\
\hline 4 & 4 & 102.519 & 100.850 & 99.374 & 105.011 & 102.590 & 104.508 \\
\hline 4 & 3 & 114.444 & 112.144 & 110.397 & 120.172 & 114.697 & 116.777 \\
\hline 4 & 2 & 139.680 & 136.190 & 133.039 & 146.380 & 139.725 & 142.574 \\
\hline 4 & 1 & 156.689 & 155.694 & 155.019 & 159.771 & 156.786 & 159.599 \\
\hline 4 & 0 & 178.699 & 177.224 & 176.246 & 190.230 & 178.984 & 182.218 \\
\hline
\end{tabular}

Notes. ${ }^{(\dagger)}$ Standard model of reference. ${ }^{(a)}$ Iron abundance profile (see Sect. 3.5.1). ${ }^{(b)}$ Envelope He/H transition profile (see Sect. 3.5.2). ${ }^{(c)}$ Nuclear reaction rates for the triple- $\alpha$ reaction (see Sect. 3.5.3).

Table 4. Parameters derived for PG 1336-018 using various iron abundance profiles.

\begin{tabular}{lcccccc}
\hline \hline Parameter & Uniform/Solar & $\frac{1}{4} \cdot \frac{N(\mathrm{Fe})}{N(\mathrm{H})}$ & $\frac{1}{2} \cdot \frac{N(\mathrm{Fe})}{N(\mathrm{H})}$ & $1 \cdot \frac{N(\mathrm{Fe})}{N(\mathrm{H})}$ & Largest drift & Likely drift \\
\hline$M / M_{\odot}$ & $0.471 \pm 0.009$ & $0.474 \pm 0.009$ & $0.474 \pm 0.009$ & $0.471 \pm 0.006$ & $+0.003 \pm 0.011$ & $+0.003 \pm 0.011$ \\
$\log g$ & $5.814 \pm 0.007$ & $5.795 \pm 0.008$ & $5.786 \pm 0.009$ & $5.775 \pm 0.007$ & $+0.039 \pm 0.010$ & $+0.020 \pm 0.011$ \\
$R / R_{\odot}$ & $0.1406 \pm 0.0011$ & $0.1443 \pm 0.0012$ & $0.1459 \pm 0.0012$ & $0.1474 \pm 0.0009$ & $-0.0068 \pm 0.0014$ & $-0.0031 \pm 0.0015$ \\
$\log q(H)$ & $-4.11 \pm 0.08$ & $-3.70 \pm 0.09$ & $-3.74 \pm 0.09$ & $-3.83 \pm 0.06$ & $-0.28 \pm 0.10$ & $+0.07 \pm 0.11$ \\
$X(C+O)$ & $0.46 \pm 0.10$ & $0.48 \pm 0.10$ & $0.51 \pm 0.09$ & $0.58 \pm 0.06$ & $-0.12 \pm 0.12$ & $-0.10 \pm 0.12$ \\
$L / L_{\odot}$ & $20.6 \pm 0.7$ & $21.8 \pm 0.7$ & $22.3 \pm 0.8$ & $22.9 \pm 0.6$ & $-2.3 \pm 0.9$ & $-1.1 \pm 0.9$ \\
$S^{2}($ opt. $)$ & 5.95 & 5.93 & 5.46 & 4.81 & & \\
$\overline{\Delta X / X}(\%)$ & 0.24 & 0.21 & 0.19 & 0.18 & & \\
$\overline{\Delta v}(\mu \mathrm{Hz})$ & 14.07 & 12.75 & 11.43 & 11.37 & & \\
\hline
\end{tabular}

diffusive equilibrium lead to a seismic solution that is the closest to the values derived for $\log g$ and $R$ from spectroscopy and orbital light curve analysis. Moreover, the best-fit solution to the observed periods is found to be noticeably better with these models, as illustrated in Table 4 where the optimal $S^{2}$ values, mean relative period (or frequency) differences $\overline{\Delta X / X}$, and mean frequency differences $\overline{\Delta v}$ achieved for the optimal fits are compared. These facts suggest that the standard models are the most realistic structures among this batch of test models for representing the oscillation properties of PG 1336-018 and that the iron profile at diffusive equilibrium is currently the approximation that is the closest to the real iron distribution in the star. We stress, however, that improving the treatment of diffusion in the next generations of sdB models in order to obtain closer fits to the observed periods and increase the precision of the measured quantities is among our long term objectives. 
V. Van Grootel et al.: Third generation stellar models for asteroseismology of hot B subdwarf stars
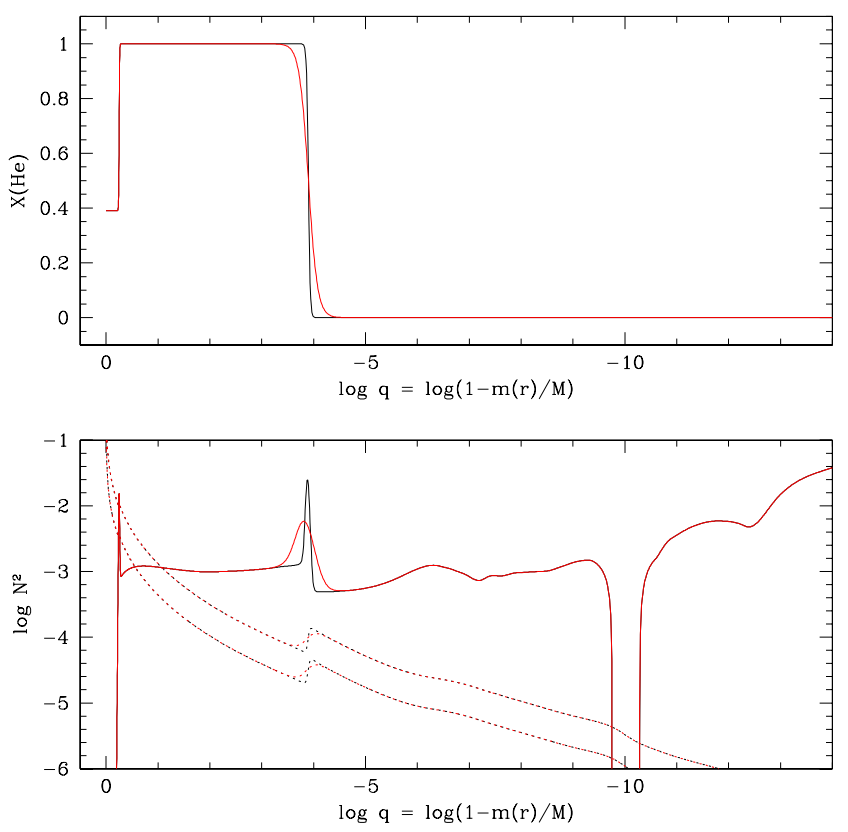

Fig. 11. Upper panel: helium abundance profiles used in two otherwise identical test models. The curves in black and red, respectively, show a sharp and a smooth $\mathrm{He} / \mathrm{H}$ transition profile at the envelope lower boundary. Lower panel: corresponding profiles for the Brunt-Väisälä (solid curves) and Lamb (dotted curves for $l=1$ and $l=2$ ) frequencies.

\subsubsection{Impact of the envelope $\mathrm{He} / \mathrm{H}$ transition profile}

As a sdB star evolves, diffusion affects the profile of the chemical transition between the H-rich envelope and the He mantle. From a relatively sharp interface at the beginning of core He-burning, the transition will slowly spread over time leading to smoother profiles (see, e.g., Hu et al. 2011)

In our static $2 \mathrm{G}$ and $3 \mathrm{G}$ models, the profile of the $\mathrm{He} / \mathrm{H}$ transition zone is controlled through a single parameter that sets the maximum slope of the composition gradient. This parameter has been calibrated based on evolutionary models from Dorman et al. (1993) that do not incorporate diffusion of helium. We therefore traditionally assume a sharp $\mathrm{He} / \mathrm{H}$ transition in our standard stellar models used for asteroseismology and the impact of this assumption on the seismic solutions is a legitimate question. We demonstrate in the experiment below that the shape of this chemical transition indeed slightly affects the periods of the relevant oscillation modes, but at a level that has no practical consequence for deriving structural parameters by asteroseismology.

We first considered two models with the same structural parameters (and our usual nonuniform iron abundance distribution from diffusive equilibrium) but with different $\mathrm{He} / \mathrm{H}$ transition profiles (see the upper panel of Fig. 11). The first model, which is the standard model of reference (see Sect. 3.3), has a sharp transition. The second model is built assuming a smooth transition. The Brunt-Väisälä and Lamb frequency profiles shown in the lower panel of Fig. 11 clearly illustrate that the differences in model structure occur exclusively in the transition region, with a wider Ledoux spike for the model having a smooth transition. The profiles remain nearly unchanged in the other regions of the star. It suggests that a rather limited impact on the pulsation periods occurs, which is confirmed in Table 3 by comparing Col. 3 and Col. 7. Between the two models, the period differences for the low-order $p$-modes of interest remain very small. It is therefore anticipated that the shape of $\mathrm{He} / \mathrm{H}$ transition zone will have
Table 5. Parameters derived for PG 1336-018 using a sharp or a smooth $\mathrm{He} / \mathrm{H}$ transition profile.

\begin{tabular}{lccc}
\hline \hline Parameter & Sharp transition & Smooth transition & Drift \\
\hline$M / M_{\odot}$ & $0.471 \pm 0.006$ & $0.476 \pm 0.009$ & $+0.005 \pm 0.011$ \\
$\log g$ & $5.775 \pm 0.007$ & $5.777 \pm 0.007$ & $+0.002 \pm 0.010$ \\
$R / R_{\odot}$ & $0.1474 \pm 0.0009$ & $0.1476 \pm 0.0011$ & $+0.0002 \pm 0.0014$ \\
$\log q(\mathrm{H})$ & $-3.83 \pm 0.06$ & $-3.79 \pm 0.08$ & $+0.04 \pm 0.10$ \\
$X(\mathrm{C}+\mathrm{O})$ & $0.58 \pm 0.06$ & $0.53 \pm 0.09$ & $-0.05 \pm 0.11$ \\
$L / L_{\odot}$ & $22.9 \pm 0.6$ & $22.8 \pm 0.7$ & $-0.1 \pm 0.9$ \\
$S^{2}($ opt. $)$ & 4.81 & 4.91 & \\
$\overline{\Delta X / X}(\%)$ & 0.18 & 0.18 & \\
$\overline{\Delta v}(\mu \mathrm{Hz})$ & 11.37 & 11.48 & \\
\hline
\end{tabular}

no significant impact on the structural parameters derived from asteroseismology.

We explicitly demonstrate the above assertion by reanalyzing PG 1336-018 with models featuring a smooth $\mathrm{He} / \mathrm{H}$ transition. The probability density functions obtained for the main structural parameters of the star are provided in Fig. B.4 (Appendix B). The corresponding statistical inferences for these parameters are given in Table 5 for comparisons with the values derived with our standard models assuming a sharp transition. No significant drift in the derived stellar parameters is observed and the achieved quality of the optimal period fit is comparable. Consequently, the effects induced by diffusion on the mantle/envelope transition profile presently have no noticeable impact on deriving the structural parameters of sdB stars by asteroseismology, considering the actual precision of the fits. This can safely be regarded as a high-order effect.

\subsubsection{Impact of nuclear reaction rates}

In a last experiment, we investigated the effects of nuclear reaction rates on the inferred parameters of the star. Our $3 \mathrm{G}$ stellar models incorporate the network of nuclear reactions (in particular the triple- $\alpha$ and related reactions for He-burning) to compute the rate of energy production that will influence the overall thermal equilibrium of the star. For our purposes, the code still relies on the Caughlan \& Fowler (1988) nuclear reaction rates. However, newer rates from Angulo et al. (1999) have since been published which, at the temperatures relevant to He-burning in sdB stars, increase by nearly $10 \%$ the triple- $\alpha$ rate and by a factor of 2 the rather uncertain ${ }^{12} \mathrm{C}(\alpha, \gamma){ }^{16} \mathrm{O}$ rate.

In order to estimate the real impact of these changes on the seismic inferences of the stellar parameters, we constructed test models where the nuclear energy production rates are increased by $10 \%$ for the triple- $\alpha$ reaction and doubled for the ${ }^{12} \mathrm{C}(\alpha, \gamma){ }^{16} \mathrm{O}$ reaction. These are referred to as the $2 \times \mathrm{CF} 88$ models. The effect of this change on the pulsation periods is given in the last column of Table 3. Compared to the model of reference, a slight nearly uniform increase of the periods is observed. This reflects the fact that for the same input parameters, the $3 \mathrm{G}$ model with the modified nuclear reaction rates leads to a star with a slightly larger radius that is, therefore, slightly less compact.

We reanalyzed PG $1336-018$ with the $2 \times$ CF88 models, and the results are given in Fig. B.5 (Appendix B) showing the probability density functions obtained for the main structural parameters of the star. The corresponding statistical inferences for these parameters are given in Table 6 for comparison with the values derived with our standard model. No significant drift in the 
Table 6. Parameters derived for PG 1336-018 using models with modified reaction rates for the triple- $\alpha$ and ${ }^{12} \mathrm{C}(\alpha, \gamma){ }^{16} \mathrm{O}$ reaction rates.

\begin{tabular}{lccc}
\hline \hline Parameter & CF88 & $2 \times \mathrm{CF} 88$ & Drift \\
\hline$M / M_{\odot}$ & $0.471 \pm 0.006$ & $0.474 \pm 0.010$ & $+0.003 \pm 0.012$ \\
$\log g$ & $5.775 \pm 0.007$ & $5.775 \pm 0.008$ & $0.00 \pm 0.011$ \\
$R / R_{\odot}$ & $0.1474 \pm 0.0009$ & $0.1479 \pm 0.0010$ & $+0.0005 \pm 0.0013$ \\
$\log q(\mathrm{H})$ & $-3.83 \pm 0.06$ & $-3.84 \pm 0.09$ & $-0.01 \pm 0.11$ \\
$X(\mathrm{C}+\mathrm{O})$ & $0.58 \pm 0.06$ & $0.54 \pm 0.10$ & $-0.04 \pm 0.12$ \\
$L / L_{\odot}$ & $22.9 \pm 0.6$ & $23.0 \pm 0.7$ & $+0.1 \pm 0.9$ \\
$S^{2}$ (opt.) & 4.81 & 5.12 & \\
$\overline{\Delta X / X}(\%)$ & 0.18 & 0.19 & \\
$\overline{\Delta v}(\mu \mathrm{Hz})$ & 11.37 & 11.52 & \\
\hline
\end{tabular}

derived stellar parameters is observed and the quality of the optimal period fit is comparable. Consequently, the impact of updating the nuclear reaction rates from Caughlan \& Fowler (1988) to Angulo et al. (1999) on the values derived from asteroseismology is not expected to be important. This will be done in future versions of our static models aimed at detailed seismic analyses of sdB stars, but clearly this is currently not a major source of uncertainty for our current purposes.

\section{Summary and conclusion}

In this paper we presented new stellar models of the socalled third generation $(3 \mathrm{G})$, aimed at performing detailed asteroseismic studies of hot B subdwarf pulsators. These models are static structures realistically representing the star from the surface to its center, contrary to the former generation of envelope models of the second generation $(2 \mathrm{G})$ that were missing the innermost regions. This improvement was a real necessity in order to exploit with asteroseismology the $g$-mode sdB pulsators that have been and are currently observed from space with CoRoT and Kepler. The new models are constructed assuming hydrostatic and thermal equilibrium for a given set of model parameters, including a chemical composition of the core. They allow for the computation of accurate periods/frequencies for the $p$-modes and the $g$-modes (including possible mixed modes), while the former generation of envelope models was limited to the $p$-modes in terms of accuracy.

To test the reliability of these new $3 \mathrm{G}$ models for asteroseismology, we reanalyzed the pulsating sdB star PG1336-018 with them. This object is a close eclipsing $\mathrm{sdB}+\mathrm{dM}$ system where the $\mathrm{sdB}$ component is a $p$-mode pulsator. This very rare configuration allows us to test some of the stellar parameters inferred from asteroseismology with the values obtained independently from the orbital light curve analysis of Vučković et al. (2007). Our new seismic results show that a very strong consistency persists at that level, as was shown to be the case with our former $2 \mathrm{G}$ models used in the seismic study of this star (Charpinet et al. 2008b). Our new analysis provides updated estimates of the structural parameters of the sdB star in PG1336-018 based on more realistic models (see Table 2). The derived parameters for the star do not differ much from those already inferred in Charpinet et al. (2008b), but more importantly they demonstrate that the asteroseismic inferences based on both $2 \mathrm{G}$ and $3 \mathrm{G}$ models are both precise and accurate, at least for the stellar parameters that could be tested, which includes the mass and radius of the star. This is an important result in view of our current attempts to construct an empirical mass distribution for comparison with predictions of various formation channels of $\mathrm{sdB}$ stars
(Fontaine et al. 2012). We also demonstrated that known model uncertainties do not affect, at the current level of accuracy, the structural parameters derived from asteroseismology. However, this is one of our long-term goals to include improved physics in stellar models, in particular the treatment of diffusion, in order to obtain closer fits to the observed pulsations and increase the precision of the derived quantities by asteroseismology.

Our approach, based on the minimization of a merit function, has been criticized in the past, arguing that the best-fit seismic model is not necessarily the most representative model of the star. We show in this analysis, through an improved treatment and estimation of the statistical significance of the inferred parameters, that the optimal model that best matches the observed periods of PG1336-018 is not an outlier of the statistical distribution of potential solutions. It can, therefore, safely be considered the model that best represents the star being studied, which is what we did in our previous analyses.

Overall, we can conclude that the main goal of this paper, which was to present and demonstrate the basic validity of the new third generation models used for asteroseismology of sdB stars, is fully achieved. Such models have passed an important test showing that they are up to this task.

Acknowledgements. This work was supported in part by the Natural Sciences and Engineering Research Council of Canada. G.F. also acknowledges the contribution of the Canada Research Chair Program. S.C. thanks the Programme National de Physique Stellaire (PNPS, CNRS/INSU, France). This work was granted access to the HPC resources of CALMIP under the allocation 2012-p0205.

\section{References}

Allard, F., Wesemael, F., Fontaine, G., Bergeron, P., \& Lamontagne, R. 1994, AJ, 107, 1565

Altmann, M., Edelmann, H., \& de Boer, K. S. 2004, A\&A, 414, 181

Angulo, C., Arnould, M., Rayet, M., et al. 1999, Nucl. Phys. A, 656, 3

Barlow, B. N., Wade, R. A., \& Liss, S. E. 2012, ApJ, 753, 101

Blanchette, J.-P., Chayer, P., Wesemael, F., et al. 2008, ApJ, 678, 1329

Böhm-Vitense, E. 1958, ZAp, 46, 108

Brassard, P., \& Fontaine, G. 2008, in Hot Subdwarf Stars and Related Objects, eds. U. Heber, C. S. Jeffery, \& R. Napiwotzki, ASP Conf. Ser., 392, 261 Brassard, P., Fontaine, G., Billères, M., et al. 2001, ApJ, 563, 1013

Brown, T. M., Ferguson, H. C., Davidsen, A. F., \& Dorman, B. 1997, ApJ, 482, 685

Busso, G., Moehler, S., Zoccali, M., Heber, U., \& Yi, S. K. 2005, ApJ, 633, L29 Caughlan, G. R., \& Fowler, W. A. 1988, At. Data Nucl. Data Tables, 40, 283 Charpinet, S. 1999, Ph.D. Thesis, Université de Montréal (Canada)

Charpinet, S., Fontaine, G., Brassard, P., \& Dorman, B. 1996, ApJ, 471, L103

Charpinet, S., Fontaine, G., Brassard, P., et al. 1997, ApJ, 483, L123

Charpinet, S., Fontaine, G., Brassard, P., \& Dorman, B. 2000, ApJS, 131, 223

Charpinet, S., Fontaine, G., \& Brassard, P. 2001, PASP, 113, 775

Charpinet, S., Fontaine, G., Brassard, P., \& Dorman, B. 2002, ApJS, 139, 487

Charpinet, S., Fontaine, G., Brassard, P., Green, E. M., \& Chayer, P. 2005, A\&A, 437, 575

Charpinet, S., Silvotti, R., Bonanno, A., et al. 2006, A\&A, 459, 565

Charpinet, S., Fontaine, G., Brassard, P., \& Chayer, P. 2008a, Commun. Asteroseismol., 157, 168

Charpinet, S., Van Grootel, V., Reese, D., et al. 2008b, A\&A, 489, 377

Charpinet, S., Fontaine, G., Brassard, P., et al. 2011a, Nature, 480, 496

Charpinet, S., Van Grootel, V., Fontaine, G., et al. 2011b, A\&A, 530, A3

Chayer, P., Fontaine, G., \& Wesemael, F. 1995, ApJS, 99, 189

D’Cruz, N. L., Dorman, B., Rood, R. T., \& O'Connell, R. W. 1996, ApJ, 466, 359

Dorman, B., Rood, R. T., \& O’Connell, R. W. 1993, ApJ, 419, 596

Fontaine, G., \& Brassard, P. 2002, ApJ, 581, L33

Fontaine, G., Graboske, H. C., Jr., \& van Horn, H. M. 1977, ApJS, 35, 293

Fontaine, G., Brassard, P., Charpinet, S., et al. 2003, ApJ, 597, 518

Fontaine, G., Brassard, P., Charpinet, S., \& Chayer, P. 2006, Mem. Soc. Astron. It., 77, 49

Fontaine, G., Brassard, P., Charpinet, S., et al. 2012, A\&A, 539, A12

Geier, S., Heber, U., Podsiadlowski, P., et al. 2010, A\&A, 519, A25

Graboske, H. C., Dewitt, H. E., Grossman, A. S., \& Cooper, M. S. 1973, ApJ, 181,457 
V. Van Grootel et al.: Third generation stellar models for asteroseismology of hot B subdwarf stars

Green, E. M., Fontaine, G., Reed, M. D., et al. 2003, ApJ, 583, L31

Green, E. M., Fontaine, G., Hyde, E. A., For, B.-Q., \& Chayer, P. 2008, in Hot Subdwarf Stars and Related Objects, eds. U. Heber, C. S. Jeffery, \& R. Napiwotzki, ASP Conf. Ser., 392, 75

Han, Z., Podsiadlowski, P., Maxted, P. F. L., Marsh, T. R., \& Ivanova, N. 2002, MNRAS, 336, 449

Han, Z., Podsiadlowski, P., Maxted, P. F. L., \& Marsh, T. R. 2003, MNRAS, 341, 669

Heber, U., Reid, I. N., \& Werner, K. 2000, A\&A, 363, 198

Hu, H., Tout, C. A., Glebbeek, E., \& Dupret, M.-A. 2011, MNRAS, 418, 195 Hubeny, I., \& Lanz, T. 1995, ApJ, 439, 875

Itoh, N., Hayashi, H., Nishikawa, A., \& Kohyama, Y. 1996, ApJS, 102, 411

Itoh, N., Uchida, S., Sakamoto, Y., Kohyama, Y., \& Nozawa, S. 2008, ApJ, 677, 495

Kilkenny, D. 2011, MNRAS, 412, 487

Kilkenny, D., Koen, C., O’Donoghue, D., \& Stobie, R. S. 1997, MNRAS, 285, 640

Kilkenny, D., O’Donoghue, D., Koen, C., Lynas-Gray, A. E., \& van Wyk, F. 1998, MNRAS, 296, 329

Kilkenny, D., Keuris, S., Marang, F., et al. 2000, The Observatory, 120, 48

Kilkenny, D., Reed, M. D., O’Donoghue, D., et al. 2003, MNRAS, 345, 834

Kitsikis, A., Fontaine, G., \& Brassard, P. 2005, in 14th European Workshop on

White Dwarfs, eds. D. Koester, \& S. Moehler, ASP Conf. Ser., 334, 65

Lamb, D. Q. 1974, Ph.D. Thesis, The University of Rochester
Lanz, T., \& Hubeny, I. 1995, ApJ, 439, 905

Lanz, T., Hubeny, I., \& Heap, S. R. 1997, ApJ, 485, 843

Maxted, P. f. L., Heber, U., Marsh, T. R., \& North, R. C. 2001, MNRAS, 326, 1391

Østensen, R. H., Green, E. M., Bloemen, S., et al. 2010, MNRAS, 408, L51

Ouazzani, R.-M., Dupret, M.-A., \& Reese, D. R. 2012, A\&A, 547, A75

Randall, S. K., Fontaine, G., Brassard, P., \& Bergeron, P. 2005, ApJS, 161, 456

Randall, S. K., Green, E. M., van Grootel, V., et al. 2007, A\&A, 476, 1317

Randall, S. K., Fontaine, G., Calamida, A., et al. 2012, in Fifth Meeting on Hot Subdwarf Stars and Related Objects, eds. D. Kilkenny, C. S. Jeffery, \& C. Koen, ASP Conf. Ser., 452, 241

Reese, D. R., MacGregor, K. B., Jackson, S., Skumanich, A., \& Metcalfe, T. S. 2009, A\&A, 506, 189

Saumon, D., Chabrier, G., \& van Horn, H. M. 1995, ApJS, 99, 713

Soker, N. 1998, AJ, 116, 1308

Tremblay, P.-E., Fontaine, G., Brassard, P., Bergeron, P., \& Randall, S. K. 2006 ApJS, 165, 551

Van Grootel, V., Charpinet, S., Fontaine, G., et al. 2008, A\&A, 488, 685

Van Grootel, V., Charpinet, S., Fontaine, G., et al. 2010a, ApJ, 718, L97

Van Grootel, V., Charpinet, S., Fontaine, G., Green, E. M., \& Brassard, P. 2010b, A\&A, 524, A63

Vučković, M., Aerts, C., Østensen, R., et al. 2007, A\&A, 471, 605

Vučković, M., Østensen, R. H., Aerts, C., et al. 2009, A\&A, 505, 239 


\section{Appendix A: Period fit and mode identification from the optimal model of PG 1336-018}

The period match and mode identification obtained for the best-fit model of PG 1336-018 are given in Table A.1. The relative and absolute differences for each pair of the $25 \mathrm{ob}-$ served/theoretical modes are provided, in period and frequency, $\Delta X / X$ (in \%), $\Delta P$ (in s), and $\Delta v$ (in $\mu \mathrm{Hz}$ ). On average, the relative dispersion between the observed and theoretical periods is $0.18 \%$, which is similar to the best-fit model of second generation uncovered by Charpinet et al. (2008b) that led to $0.17 \%$.

Independent constraints on the mode identification in PG 1336-018 exist from time-resolved spectroscopy (Vučković et al. 2009). Their detailed line-profile analysis excluded the $(\ell,|m|)=(3,3),(1,0),(2,1)$, or $(2,0)$ modes for the $5435 \mu \mathrm{Hz}$ pulsation $\left(f_{5}\right.$ in Table A.1), although data were too noisy to uniquely identify this pulsation with the radial or sectoral dipole/quadrupole modes. The $5435 \mu \mathrm{Hz} f_{5}$ pulsation is identified as the fundamental radial mode $(k, \ell, m)=(0,0,0)$ in Table A.1, which is consistent with the results of Vučković et al. 2009 (see in particular their Fig. 13).

It is also interesting to look back at the three uncertain periods of Kilkenny et al. (2003) that were not included in the seismic search, $184.04 \mathrm{~s}\left(f_{10}\right), 178.96 \mathrm{~s}\left(f_{13}\right)$, and $173.59 \mathrm{~s}\left(f_{8}\right)$. The periods $f_{10}$ and $f_{8}$ all have an acceptable counterpart in the best-fit model theoretical spectrum (see Table A.1). The period $f_{10}$ can be associated with the $(l, k, m)=(2,0,-1)$ mode for a relative dispersion $\Delta X / X=-0.33 \%$ and $f_{8}$ finds a counterpart at $\Delta X / X=-0.26 \%$ with the $(l, k, m)=(4,0,-2)$ mode. These matches do not significantly affect the overall quality of the fit. A $l=3$ mode (not shown here) is needed to account for the last uncertain period, $178.96 \mathrm{~s}\left(f_{13}\right)$. We recall, however, that the existence of these periods needs to be confirmed by additional observations.

The mode identification of the best-fit model respects quite well, qualitatively, the expected amplitude hierarchy. On average, one may expect that modes of higher $l$ would have lower apparent amplitudes even though this argument can hardly be applied to individual identifications, since intrinsic amplitudes are not known and may vary significantly from one mode to another. According to the mode identification in Table A.1, the observed average amplitudes $\overline{A_{l}}$ are $\overline{A_{0}}=0.145 \%$ (for $l=0$ modes), $\overline{A_{1}}=0.230 \%, \overline{A_{2}}=0.183 \%$, and $\overline{A_{4}}=0.081 \%$. This significant drop for $l=4$ modes corresponds well to the theoretical expectation that modes with $l=4$ have a much lower visibility than the $l \leq 2$ modes (Randall et al. 2005, 2007). 
V. Van Grootel et al.: Third generation stellar models for asteroseismology of hot B subdwarf stars

Table A.1. Mode identification and details of the frequency fit obtained for the optimal solution.

\begin{tabular}{|c|c|c|c|c|c|c|c|c|c|c|c|}
\hline$l$ & $k$ & $m$ & $\begin{array}{c}\begin{array}{c}v_{\mathrm{obs}} \\
(\mu \mathrm{Hz})\end{array} \\
\end{array}$ & $\begin{array}{c}v_{\text {th }} \\
(\mu \mathrm{Hz})\end{array}$ & $\begin{array}{c}P_{\text {obs }} \\
(\mathrm{s})\end{array}$ & $\begin{array}{l}P_{\text {th }} \\
(\mathrm{s})\end{array}$ & $\begin{array}{c}\Delta X / X \\
(\%)\end{array}$ & $\begin{array}{l}\Delta P \\
(\mathrm{~s})\end{array}$ & $\begin{array}{c}\Delta v \\
(\mu \mathrm{Hz})\end{array}$ & $\begin{array}{c}\text { Amplitude } \\
(\%)\end{array}$ & Id. \\
\hline 0 & 2 & 0 & $\ldots$ & 6849.952 & & 145.99 & & & & & \\
\hline 0 & 1 & 0 & 5891.363 & 5896.601 & 169.74 & 169.59 & +0.09 & +0.15 & -5.238 & 0.0900 & $f_{15}$ \\
\hline 0 & 0 & 0 & 5435.373 & 5431.524 & 183.98 & 184.11 & -0.07 & -0.13 & +3.849 & 0.2000 & $f_{5}$ \\
\hline 1 & 3 & -1 & & 7249.631 & & $\begin{array}{l}137.94 \\
\end{array}$ & 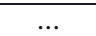 & & & & \\
\hline 1 & 3 & 0 & 7108.836 & 7138.460 & 140.67 & 140.09 & +0.41 & +0.58 & -29.623 & 0.0900 & $f_{14}$ \\
\hline 1 & 3 & +1 & $\ldots$ & 7027.288 & $\ldots$ & 142.30 & $\ldots$ & $\ldots$ & $\ldots$ & & \\
\hline 1 & 2 & -1 & $\ldots$ & 6721.396 & $\ldots$ & 148.78 & $\ldots$ & $\ldots$ & $\ldots$ & $\ldots$ & \\
\hline 1 & 2 & 0 & $\ldots$ & 6609.834 & $\ldots$ & 151.29 & $\ldots$ & $\ldots$ & $\ldots$ & $\ldots$ & \\
\hline 1 & 2 & +1 & $\ldots$ & 6498.272 & $\ldots$ & 153.89 & $\ldots$ & $\ldots$ & $\ldots$ & $\ldots$ & \\
\hline 1 & 1 & -1 & 5585.656 & 5581.761 & 179.03 & 179.15 & -0.07 & -0.12 & +3.895 & 0.4000 & $f_{2}$ \\
\hline 1 & 1 & 0 & 5470.759 & 5469.172 & 182.79 & 182.84 & -0.03 & -0.05 & +1.587 & 0.0600 & $f_{22}$ \\
\hline 1 & 1 & +1 & 5369.416 & 5356.584 & 186.24 & 186.69 & -0.24 & -0.45 & +12.832 & 0.3700 & $f_{3}$ \\
\hline 2 & 3 & -2 & $\ldots$ & 8079.349 & $\ldots$ & 123.77 & $\ldots$ & $\ldots$ & $\ldots$ & $\ldots$ & \\
\hline 2 & 3 & -1 & 7948.494 & 7976.309 & 125.81 & 125.37 & +0.35 & +0.44 & -27.815 & 0.0600 & $f_{24}$ \\
\hline 2 & 3 & 0 & 7880.842 & 7873.268 & 126.89 & 127.01 & -0.10 & -0.12 & +7.573 & 0.0700 & $f_{19}$ \\
\hline 2 & 3 & +1 & $\ldots$ & 7770.228 & $\ldots$ & 128.70 & $\ldots$ & $\ldots$ & $\ldots$ & $\ldots$ & \\
\hline 2 & 3 & +2 & $\ldots$ & 7667.188 & $\ldots$ & 130.43 & $\ldots$ & $\ldots$ & $\ldots$ & $\ldots$ & \\
\hline 2 & 2 & -2 & $\ldots$ & 7051.422 & $\ldots$ & 141.82 & $\ldots$ & $\ldots$ & $\ldots$ & $\ldots$ & \\
\hline 2 & 2 & -1 & $\ldots$ & 6940.368 & $\ldots$ & 144.08 & $\ldots$ & $\ldots$ & $\ldots$ & $\ldots$ & \\
\hline 2 & 2 & 0 & $\ldots$ & 6829.314 & $\ldots$ & 146.43 & $\ldots$ & $\ldots$ & $\ldots$ & $\ldots$ & \\
\hline 2 & 2 & +1 & $\ldots$ & 6718.259 & $\ldots$ & 148.85 & $\ldots$ & $\ldots$ & $\ldots$ & $\ldots$ & \\
\hline 2 & 2 & +2 & $\ldots$ & 6607.205 & $\ldots$ & 151.35 & $\ldots$ & $\ldots$ & $\ldots$ & $\ldots$ & \\
\hline 2 & 1 & -2 & 5757.384 & 5785.532 & 173.69 & 172.84 & +0.49 & +0.84 & -28.148 & 0.4700 & $f_{1}$ \\
\hline 2 & 1 & -1 & $\ldots$ & 5686.870 & $\ldots$ & 175.84 & $\ldots$ & $\ldots$ & $\ldots$ & $\ldots$ & \\
\hline 2 & 1 & 0 & 5598.477 & 5588.209 & 178.62 & 178.95 & -0.18 & -0.33 & +10.268 & 0.1700 & $f_{6}$ \\
\hline 2 & 1 & +1 & $\ldots$ & 5489.548 & $\ldots$ & 182.16 & $\ldots$ & $\ldots$ & $\ldots$ & $\ldots$ & \\
\hline 2 & 1 & +2 & 5392.289 & 5390.887 & 185.45 & 185.50 & -0.03 & -0.05 & +1.402 & 0.2500 & $f_{4}$ \\
\hline 2 & 0 & -2 & 5505.698 & 5504.174 & 181.63 & 181.68 & -0.03 & -0.05 & +1.525 & 0.0800 & $f_{17}$ \\
\hline 2 & 0 & -1 & $\ldots$ & 5416.040 & [184.04] & 184.64 & $\ldots$ & $\ldots$ & $\ldots$ & $\ldots$ & {$\left[f_{10}\right]$} \\
\hline 2 & 0 & 0 & $\ldots$ & 5327.907 & $\ldots$ & 187.69 & $\ldots$ & $\ldots$ & $\ldots$ & $\ldots$ & \\
\hline 2 & 0 & +1 & $\ldots$ & 5239.773 & $\ldots$ & 190.85 & $\ldots$ & $\ldots$ & $\ldots$ & $\ldots$ & \\
\hline 2 & 0 & +2 & $\ldots$ & 5151.640 & $\ldots$ & 194.11 & $\ldots$ & $\ldots$ & $\ldots$ & $\ldots$ & \\
\hline 4 & 5 & -4 & $\ldots$ & 11203.734 & $\ldots$ & 89.26 & $\ldots$ & $\ldots$ & $\ldots$ & $\ldots$ & \\
\hline 4 & 5 & -3 & $\ldots$ & 11092.302 & $\ldots$ & 90.15 & $\ldots$ & $\ldots$ & $\ldots$ & $\ldots$ & \\
\hline 4 & 5 & -2 & $\ldots$ & 10980.871 & $\ldots$ & 91.07 & $\ldots$ & $\ldots$ & $\ldots$ & $\ldots$ & \\
\hline 4 & 5 & -1 & $\ldots$ & 10869.439 & $\ldots$ & 92.00 & $\ldots$ & $\ldots$ & $\ldots$ & $\ldots$ & \\
\hline 4 & 5 & 0 & $\ldots$ & 10758.007 & $\ldots$ & 92.95 & $\ldots$ & $\ldots$ & $\ldots$ & $\ldots$ & \\
\hline 4 & 5 & +1 & $\ldots$ & 10646.576 & $\ldots$ & 93.93 & $\ldots$ & $\ldots$ & $\ldots$ & $\ldots$ & \\
\hline 4 & 5 & +2 & $\ldots$ & 10535.144 & $\ldots$ & 94.92 & $\ldots$ & $\ldots$ & $\ldots$ & $\ldots$ & \\
\hline 4 & 5 & +3 & $\ldots$ & 10423.713 & $\ldots$ & 95.94 & $\ldots$ & $\ldots$ & $\ldots$ & $\ldots$ & \\
\hline 4 & 5 & +4 & 10314.595 & 10312.281 & 96.95 & 96.97 & -0.02 & -0.02 & +2.314 & 0.0500 & $f_{26}$ \\
\hline$\cdots$ & $\cdots$ & $\cdots$ & $\cdots$ & $\cdots$ & $\cdots$ & $\cdots$ & $\cdots$ & $\cdots$ & $\cdots$ & $\cdots$ & \\
\hline 4 & 2 & -4 & $\ldots$ & 7495.823 & $\ldots$ & 133.41 & $\ldots$ & $\ldots$ & $\ldots$ & $\ldots$ & \\
\hline 4 & 2 & -3 & 7412.898 & 7386.019 & 134.90 & 135.39 & -0.36 & -0.49 & +26.880 & 0.0600 & $f_{23}$ \\
\hline 4 & 2 & -2 & $\ldots$ & 7276.214 & $\ldots$ & 137.43 & $\ldots$ & $\ldots$ & $\ldots$ & $\ldots$ & \\
\hline 4 & 2 & -1 & $\ldots$ & 7166.409 & $\ldots$ & 139.54 & $\ldots$ & $\ldots$ & $\ldots$ & $\ldots$ & \\
\hline 4 & 2 & 0 & 7071.136 & 7056.605 & 141.42 & 141.71 & -0.21 & -0.29 & +14.531 & 0.1300 & $f_{9}$ \\
\hline 4 & 2 & +1 & $\ldots$ & 6946.800 & $\ldots$ & 143.95 & $\ldots$ & $\ldots$ & $\ldots$ & $\ldots$ & \\
\hline 4 & 2 & +2 & $\ldots$ & 6836.995 & $\ldots$ & 146.26 & $\ldots$ & $\ldots$ & $\ldots$ & $\ldots$ & \\
\hline 4 & 2 & +3 & $\ldots$ & 6727.191 & $\ldots$ & 148.65 & $\ldots$ & $\ldots$ & $\ldots$ & $\ldots$ & \\
\hline 4 & 2 & +4 & $\ldots$ & 6617.386 & $\ldots$ & 151.12 & $\ldots$ & $\cdots$ & $\cdots$ & $\cdots$ & \\
\hline 4 & 1 & -4 & $\ldots$ & 6726.790 & $\ldots$ & 148.66 & $\ldots$ & $\ldots$ & $\ldots$ & $\ldots$ & \\
\hline 4 & 1 & -3 & $\ldots$ & 6626.935 & $\ldots$ & 150.90 & $\ldots$ & $\ldots$ & $\ldots$ & $\ldots$ & \\
\hline 4 & 1 & -2 & $\ldots$ & 6527.080 & $\ldots$ & 153.21 & $\ldots$ & $\ldots$ & $\ldots$ & $\ldots$ & \\
\hline 4 & 1 & -1 & $\ldots$ & 6427.225 & $\ldots$ & 155.59 & $\ldots$ & $\ldots$ & $\ldots$ & $\ldots$ & \\
\hline
\end{tabular}

Notes. The mean relative dispersion of the fit is $\overline{\Delta X / X}=0.18 \%$ (or $\overline{\Delta P}=0.30 \mathrm{~s}$ and $\overline{\Delta v}=11.366 \mu \mathrm{Hz} ; S^{2}=4.812$ ). 
A\&A 553, A97 (2013)

Table A.1. continued.

\begin{tabular}{|c|c|c|c|c|c|c|c|c|c|c|c|}
\hline$l$ & $k$ & $m$ & $\begin{array}{c}v_{\mathrm{obs}} \\
(\mu \mathrm{Hz})\end{array}$ & $\begin{array}{c}v_{\text {th }} \\
(\mu \mathrm{Hz})\end{array}$ & $\begin{array}{c}P_{\text {obs }} \\
(\mathrm{s})\end{array}$ & $\begin{array}{l}P_{\text {th }} \\
(\mathrm{s})\end{array}$ & $\begin{array}{c}\Delta X / X \\
(\%)\end{array}$ & $\begin{array}{l}\Delta P \\
(\mathrm{~s})\end{array}$ & $\begin{array}{c}\Delta v \\
(\mu \mathrm{Hz})\end{array}$ & $\begin{array}{c}\text { Amplitude } \\
(\%)\end{array}$ & Id. \\
\hline 4 & 1 & 0 & $\ldots$ & 6327.371 & $\ldots$ & 158.04 & $\ldots$ & $\ldots$ & $\ldots$ & $\ldots$ & \\
\hline 4 & 1 & +1 & $\ldots$ & 6227.516 & $\ldots$ & 160.58 & ... & $\ldots$ & & $\ldots$ & \\
\hline 4 & 1 & +2 & 6163.328 & 6127.661 & 162.25 & 163.19 & -0.58 & -0.94 & +35.667 & 0.0500 & $f_{28}$ \\
\hline 4 & 1 & +3 & $\ldots$ & 6027.806 & $\ldots$ & 165.90 & $\ldots$ & $\ldots$ & . & $\ldots$ & \\
\hline 4 & 1 & +4 & 5916.110 & 5927.951 & 169.03 & 168.69 & +0.20 & +0.34 & -11.842 & 0.1000 & $f_{11}$ \\
\hline 4 & 0 & -4 & $\ldots$ & 5969.956 & $\ldots$ & 167.51 & $\ldots$ & $\ldots$ & $\ldots$ & $\ldots$ & \\
\hline 4 & 0 & -3 & $\ldots$ & 5857.737 & $\ldots$ & 170.71 & $\ldots$ & $\ldots$ & $\ldots$ & $\ldots$ & \\
\hline 4 & 0 & -2 & $\ldots$ & 5745.518 & [173.59] & 174.05 & $\ldots$ & $\ldots$ & . & $\ldots$ & {$\left[f_{8}\right]$} \\
\hline 4 & 0 & -1 & 5621.135 & 5633.299 & 177.90 & 177.52 & +0.22 & +0.38 & -12.164 & 0.0700 & $f_{20}$ \\
\hline 4 & 0 & 0 & 5516.633 & 5521.080 & 181.27 & 181.12 & +0.08 & +0.15 & -4.447 & 0.1000 & $f_{12}$ \\
\hline 4 & 0 & +1 & 5401.026 & 5408.861 & 185.15 & 184.88 & +0.14 & +0.27 & -7.835 & 0.0600 & $f_{25}$ \\
\hline 4 & 0 & +2 & $\ldots$ & 5296.642 & $\ldots$ & 188.80 & $\ldots$ & $\ldots$ & $\ldots$ & $\ldots$ & \\
\hline 4 & 0 & +3 & $\ldots$ & 5184.423 & $\ldots$ & 192.89 & $\ldots$ & $\ldots$ & $\ldots$ & $\ldots$ & \\
\hline 4 & 0 & +4 & $\ldots$ & 5072.205 & ... & 197.15 & ... & $\ldots$ & ... & $\ldots$ & \\
\hline 4 & -1 & -4 & 5444.251 & 5450.925 & 183.68 & 183.46 & +0.12 & +0.22 & -6.674 & 0.1700 & $f_{7}$ \\
\hline 4 & -1 & -3 & 5356.473 & 5338.149 & 186.69 & 187.33 & -0.34 & -0.64 & +18.324 & 0.0800 & $f_{16}$ \\
\hline 4 & -1 & -4 & 5218.934 & 5225.373 & 191.61 & 191.37 & +0.12 & +0.24 & -6.439 & 0.0600 & $f_{21}$ \\
\hline 4 & -1 & -1 & 5111.168 & 5112.598 & 195.65 & 195.60 & +0.03 & +0.05 & -1.430 & 0.0500 & $f_{27}$ \\
\hline 4 & -1 & 0 & $\ldots$ & 4999.822 & $\ldots$ & 200.01 & $\ldots$ & $\ldots$ & $\ldots$ & $\ldots$ & \\
\hline 4 & -1 & +1 & 4885.198 & 4887.046 & 204.70 & 204.62 & +0.04 & +0.08 & -1.848 & 0.0700 & $f_{18}$ \\
\hline 4 & -1 & +2 & $\ldots$ & 4774.270 & $\ldots$ & 209.46 & $\ldots$ & $\ldots$ & $\ldots$ & $\ldots$ & \\
\hline 4 & -1 & +3 & $\ldots$ & 4661.494 & $\ldots$ & 214.52 & $\ldots$ & $\ldots$ & $\ldots$ & $\ldots$ & \\
\hline 4 & -1 & +4 & $\ldots$ & 4548.718 & $\ldots$ & 219.84 & $\ldots$ & $\ldots$ & $\ldots$ & $\ldots$ & \\
\hline
\end{tabular}


V. Van Grootel et al.: Third generation stellar models for asteroseismology of hot B subdwarf stars

\section{Appendix B: Probability density functions of stellar parameters with known model uncertainties}


Fig. B.1. Probability density functions derived from asteroseismology using models with a uniform distribution of iron in solar proportions. The parameters inferred for the sdB component of PG 1336-018 are, from the upper-left panel to the lower-right panel, the mass $M$, log $g$, the radius $R, \log q(\mathrm{H}), \log q($ core $), X_{\text {core }}(\mathrm{C}+\mathrm{O})$, and $L_{*} / L_{\odot}$. The red-hatched regions between the two vertical solid red lines shows the $1 \sigma$ range containing $68.3 \%$ of the distribution. In the panel showing the mass, the filled circles with the dotted lines are the three orbital solutions for the mass of the sdB component proposed by Vučković et al. (2007) with their $1 \sigma$ uncertainties. In the panel showing $\log g$, The filled circle with the solid line and dotted line indicate the value derived from spectroscopy with its $1 \sigma$ and $3 \sigma$ uncertainties, respectively. Finally, in the panel showing the radius, the filled circle with the solid horizontal line indicates the $1 \sigma$ error associated with the relevant orbital solution from Vučković et al. (2007). In each panel, the blue vertical dashed and dotted lines show the seismic solution and its $1 \sigma$ uncertainty obtained with our standard $3 \mathrm{G}$ models. 
A\&A 553, A97 (2013)
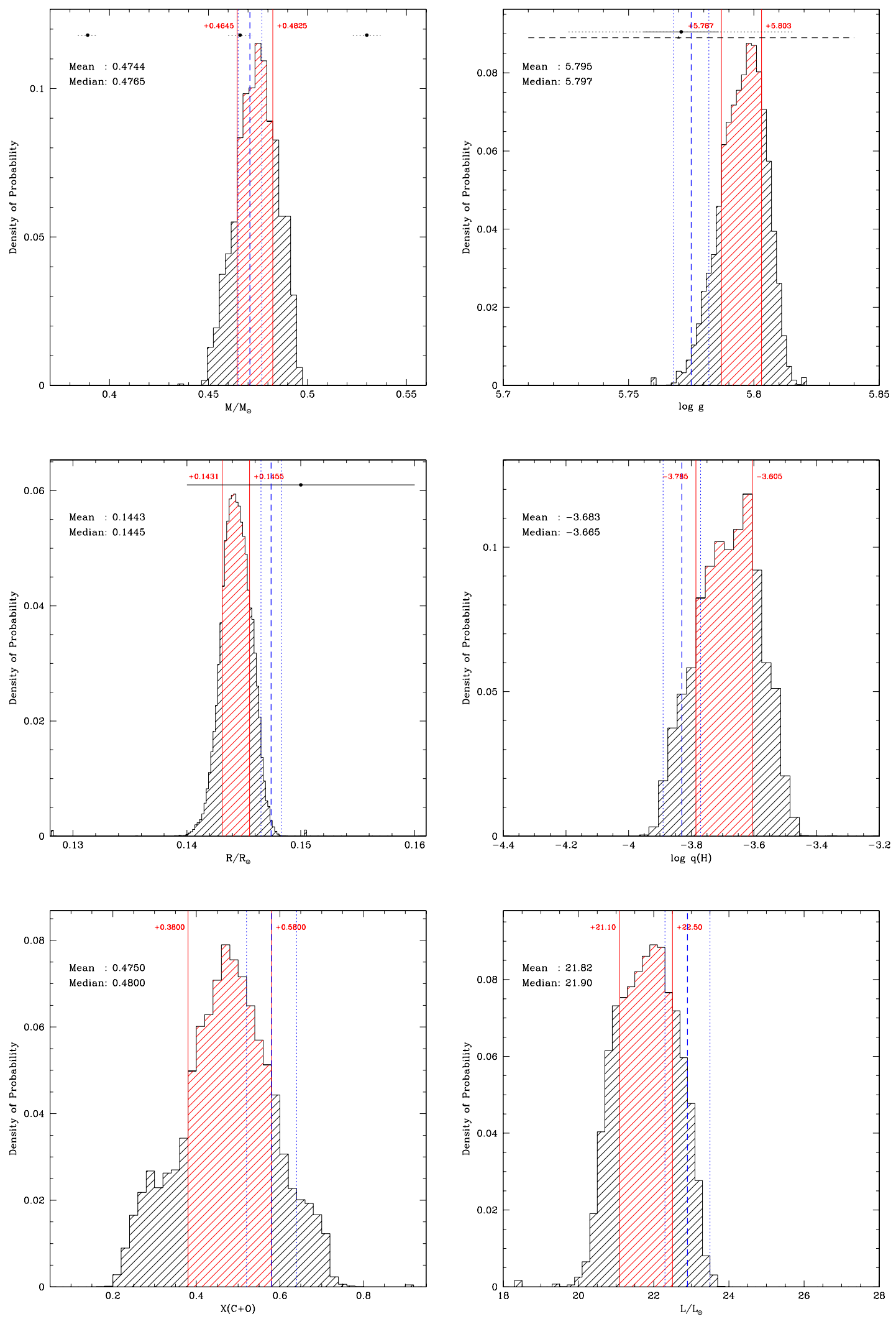

Fig. B.2. Same as Fig. B.1, but using models with a nonuniform iron abundance profile reduced by a factor of four relative to the value expected at diffusive equilibrium. 
V. Van Grootel et al.: Third generation stellar models for asteroseismology of hot B subdwarf stars
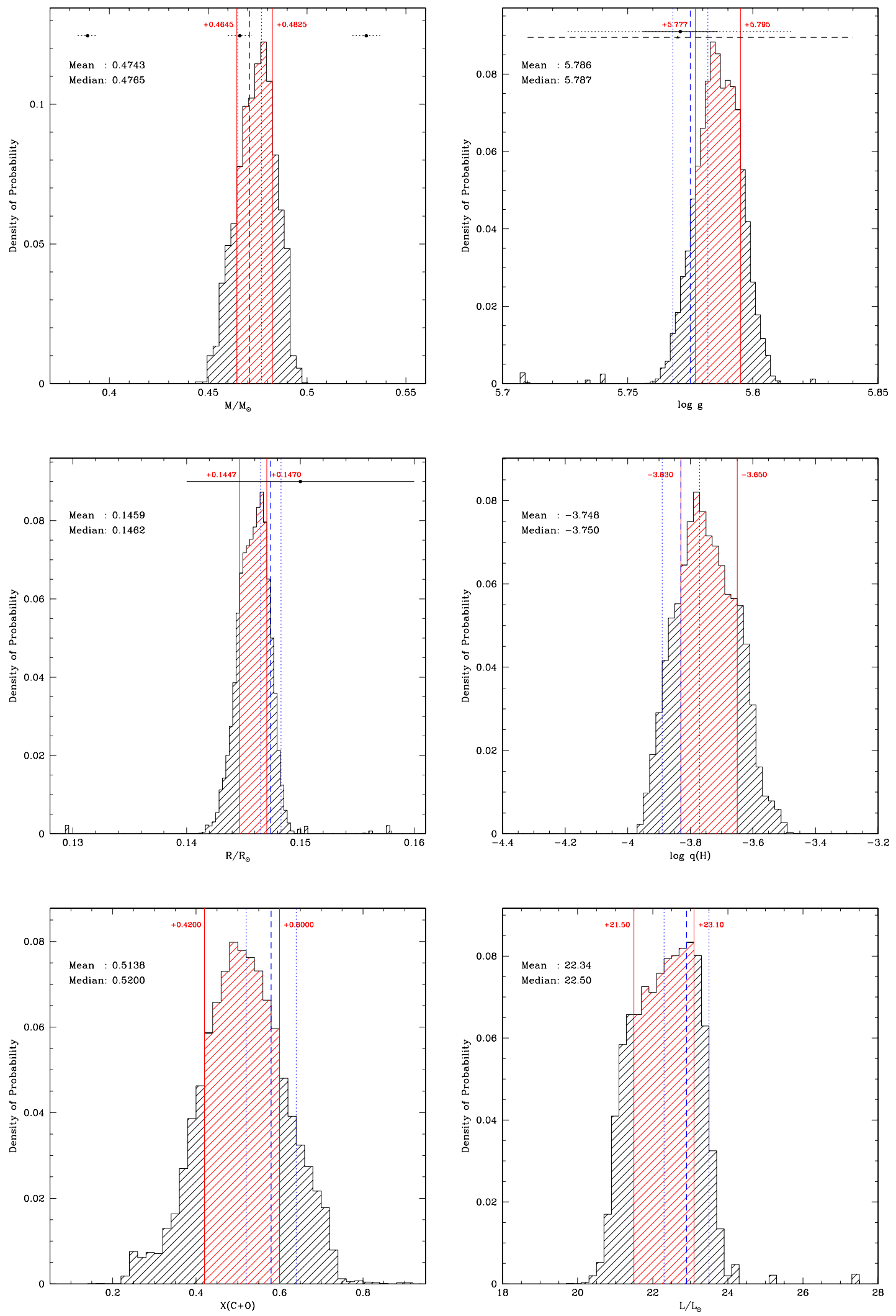

Fig. B.3. Same as Fig. B.1, but using models with a nonuniform iron abundance profile reduced by a factor of two relative to the value expected at diffusive equilibrium. 
A\&A 553, A97 (2013)
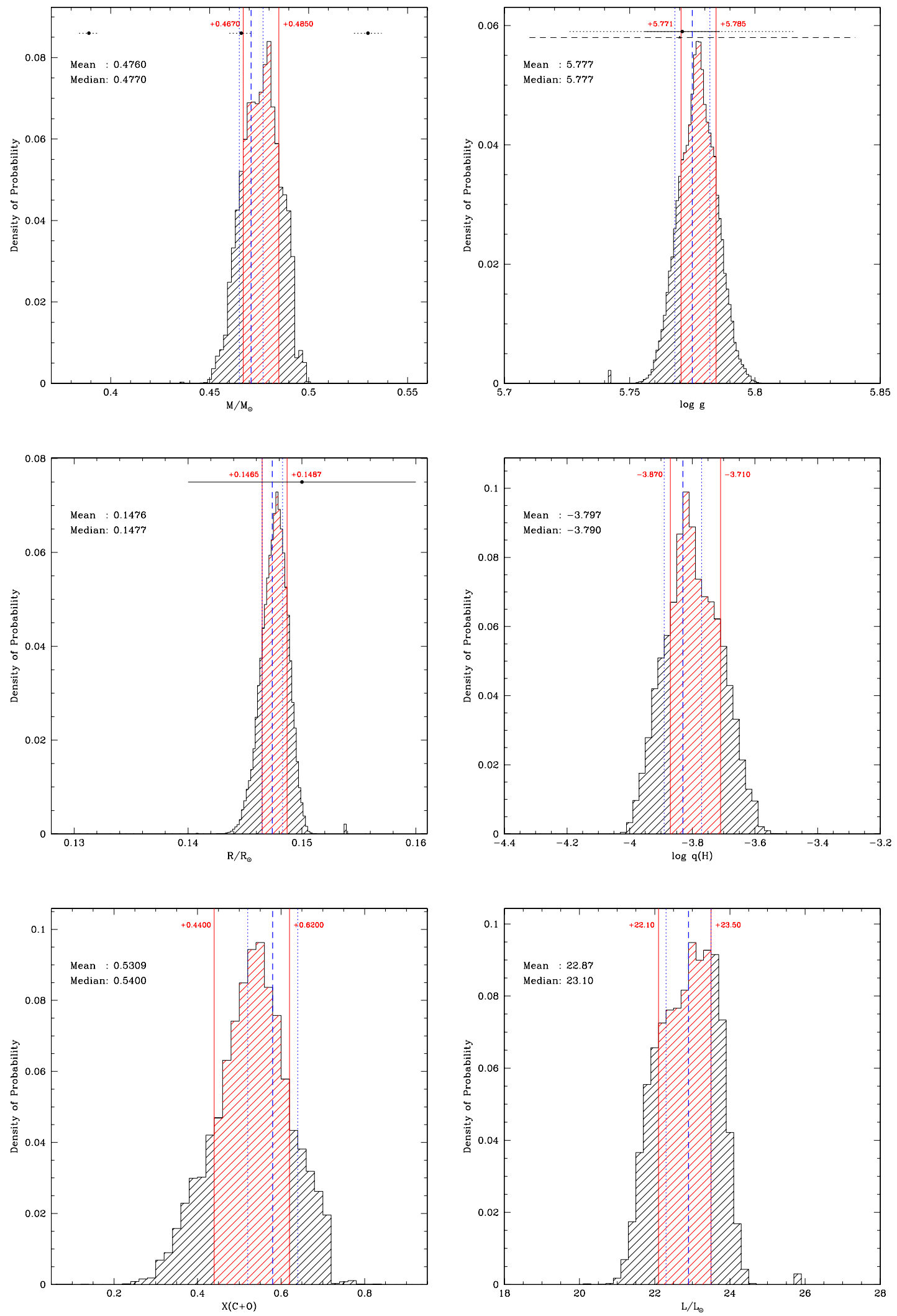

Fig. B.4. Same as Fig. B.1, but using models with a smooth $\mathrm{He} / \mathrm{H}$ transition. 
V. Van Grootel et al.: Third generation stellar models for asteroseismology of hot B subdwarf stars
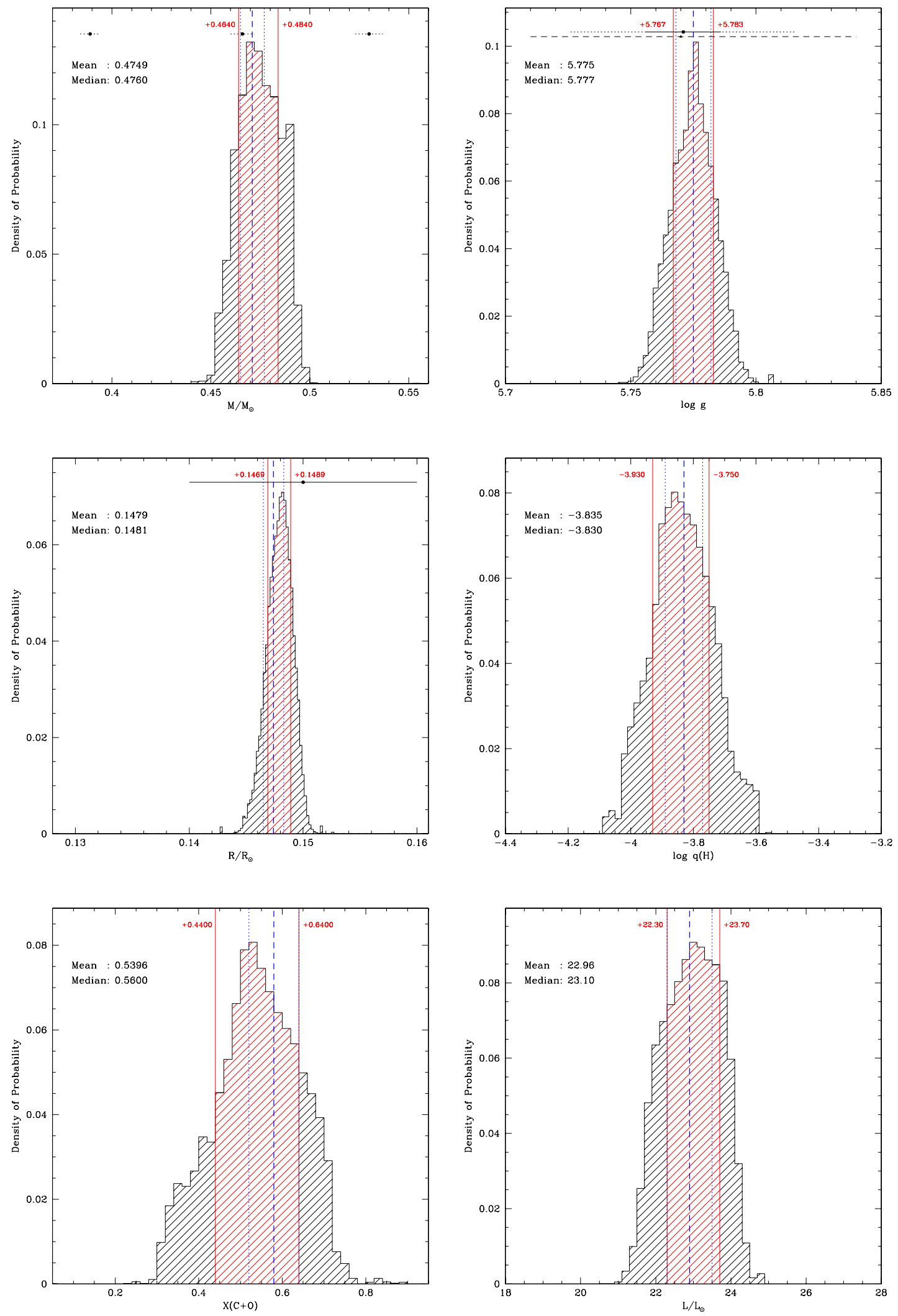

Fig. B.5. Same as Fig. B.1, but using models with modified rates for the triple- $\alpha$ and ${ }^{12} \mathrm{C}(\alpha, \gamma){ }^{16} \mathrm{O}$ nuclear reactions. 